\title{
A new hybrid general iterative algorithm for common solutions of generalized mixed equilibrium problems and variational inclusions
}

\author{
Kriengsak Wattanawitoon ${ }^{1}$, Thanyarat Jitpeera ${ }^{2}$ and Poom Kumam²
}

\author{
* Correspondence: poom. \\ kum@kmutt.ac.th \\ ${ }^{2}$ Department of Mathematics, \\ Faculty of Science, King Mongkut's \\ University of Technology (KMUTT), \\ Bangmod, Thrungkru, Bangkok \\ 10140, Thailand \\ Full list of author information is \\ available at the end of the article
}

\begin{abstract}
In this article, we introduce a new general iterative method for finding a common element of the set of solutions generalized for mixed equilibrium problems, the set of solution for fixed point for nonexpansive mappings and the set of solutions for the variational inclusions for $\beta_{1}, \beta_{2}$-inverse-strongly monotone mappings in a real Hilbert space. We prove that the sequence converges strongly to a common element of the above sets under some suitable conditions. Our results improve and extend the corresponding results of Marino and Xu, Su et al., Tan and Chang and some authors.

Mathematics subject Classification: 46C05; 47H09; 47H10.
\end{abstract}

Keywords: nonexpansive mapping, inverse-strongly monotone mapping, generalized mixed equilibrium problem, variational inclusion

\section{Introduction}

Let $C$ be a closed convex subset of a real Hilbert space $H$ with the inner product 〈., .) and the norm $\|\cdot\|$. Let $F$ be a bifunction of $C \times C$ into $\mathcal{R}$, where $\mathcal{R}$ is the set of real numbers, $\Psi: C \rightarrow H$ be a mapping and $\varphi: C \rightarrow \mathcal{R}$ be a real-valued function. The generalized mixed equilibrium problem for finding $x \in C$ such that

$$
F(x, y)+\langle\Psi x, y-x\rangle+\varphi(y)-\varphi(x) \geq 0, \quad \forall y \in C
$$

The set of solutions of (1.1) is denoted by $\operatorname{GMEP}(F, \phi, \Psi)$, that is

$$
\operatorname{GMEP}(F, \varphi, \Psi)=\{x \in C: F(x, y)+\langle\Psi x, y-x\rangle+\varphi(y)-\varphi(x) \geq 0, \quad \forall y \in C\}
$$

If $F \equiv 0$, the problem (1.1) is reduced into the mixed variational inequality of Browder type [1] for finding $x \in C$ such that

$$
\langle\Psi x, y-x\rangle+\varphi(y)-\varphi(x) \geq 0, \quad \forall y \in C
$$

The set of solutions of (1.2) is denoted by $\operatorname{MVI}(C, \phi, \Psi)$.

If $\Psi \equiv 0$, the problem (1.1) is reduced into the mixed equilibrium problem for finding $x \in C$ such that

$$
F(x, y)+\varphi(y)-\varphi(x) \geq 0, \quad \forall y \in C
$$

\section{Springer}

(c) 2012 Wattanawitoon et al; licensee Springer. This is an Open Access article distributed under the terms of the Creative Commons Attribution License (http://creativecommons.org/licenses/by/2.0), which permits unrestricted use, distribution, and reproduction in any medium, provided the original work is properly cited. 
The set of solutions of (1.3) is denoted by $\operatorname{MEP}(F, \phi)$.

If $\phi \equiv 0$, the problem (1.3) is reduced into the equilibrium problem [2] for finding $x \in C$ such that

$$
F(x, y) \geq 0, \quad \forall y \in C .
$$

The set of solutions of (1.4) is denoted by $\operatorname{EP}(F)$. See, e.g. [3-6] and the references therein.

If $F \equiv 0$ and $\phi \equiv 0$, the problem (1.1) is reduced into the Hartmann-Stampacchia variational inequality [7] for finding $x \in C$ such that

$$
\langle\Psi x, y-x\rangle \geq 0, \quad \forall y \in C .
$$

The set of solutions of (1.5) is denoted by $\operatorname{VI}(C, \Psi)$.

If $F \equiv 0$ and $\Psi \equiv 0$, the problem (1.1) is reduced into the minimize problem for finding $x \in C$ such that

$$
\varphi(y)-\varphi(x) \geq 0, \quad \forall y \in C .
$$

The set of solutions of (1.6) is denoted by $\operatorname{Argmin}(\phi)$.

Iterative methods for nonexpansive mappings have recently been applied to solve convex minimization problems. Convex minimization problems have a great impact and influence in the development of almost all branches of pure and applied sciences. A typical problem is to minimize a quadratic function over the set of the fixed points of a nonexpansive mapping on a real Hilbert space $H$ :

$$
\theta(x)=\frac{1}{2}\langle A x, x\rangle-\langle x, y\rangle, \quad \forall x \in F(S),
$$

where $A$ is a linear bounded operator, $F(S)$ is the fixed point set of a nonexpansive mapping $S$ and $y$ is a given point in $H$ [8].

Recall, a mapping $S: C \rightarrow C$ is said to be nonexpansive if

$$
\|S x-S y\| \leq\|x-y\|, \quad \forall x, y \in C .
$$

If $C$ is bounded closed convex and $S$ is a nonexpansive mapping of $C$ into itself, then $F(S)$ is nonempty [9]. A mapping $S: C \rightarrow C$ is said to be a $k$-strictly pseudo-contraction [10] if there exists $0 \leq k<1$ such that

$$
\|S x-S y\|^{2} \leq\|x-y\|^{2}+k\|(I-S) x-(I-S) y\|^{2}, \quad \forall x, y \in C,
$$

where $I$ denotes the identity operator on $C$. A mapping $A$ of $C$ into $H$ is called monotone if

$$
\langle A x-A y, x-y\rangle \geq 0, \quad \forall x, y \in C .
$$

A mapping $A$ of $C$ into $H$ is called an $\alpha$-inverse-strongly monotone if there exists a positive real number $\alpha$ such that

$$
\langle A x-A y, x-y\rangle \geq \alpha\|A x-A y\|^{2}, \quad \forall x, y \in C .
$$

A mapping $A$ of $C$ into $H$ is called $\alpha$-strongly monotone if there exists a positive real number $\alpha$ such that 


$$
\langle A x-A y, x-y\rangle \geq \alpha\|x-y\|^{2}, \quad \forall x, y \in C .
$$

A linear bounded operator $A$ is called strongly positive if there exists a constant $\bar{\gamma}>0$ with the property

$$
\langle A x, x\rangle \geq \bar{\gamma}\|x\|^{2}, \quad \forall x \in H .
$$

A self mapping $f: C \rightarrow C$ is called contraction on $C$ if there exists a constant $\alpha \in(0$, 1) such that

$$
\|f(x)-f(y)\| \leq \alpha\|x-y\|, \quad \forall x, y \in C .
$$

Let $B: H \rightarrow H$ be a single-valued nonlinear mapping and $M: H \rightarrow 2^{H}$ be a setvalued mapping. The variational inclusion problem is to find $x \in H$ such that

$$
\theta \in B(x)+M(x),
$$

where $\theta$ is the zero vector in $H$. The set of solutions of problem (1.8) is denoted by $I$ $(B, M)$. The variational inclusion has been extensively studied in the literature. See, e.g. [11-15] and the reference therein.

A set-valued mapping $M: H \rightarrow 2^{H}$ is called monotone if for all $x, y \in H, f \in M(x)$ and $g \in M(y)$ imply $\langle x-y, f-g\rangle \geq 0$. A monotone mapping $M$ is maximal if its graph $G(M):=\{(f, x) \in H \times H: f \in M(x)\}$ of $M$ is not properly contained in the graph of any other monotone mapping. It is known that a monotone mapping $M$ is maximal if and only if for $(x, f) \in H \times H,\langle x-y, f-g\rangle>0$ for all $(y, g) \in G(M)$ imply $f \in M$ $(x)$.

Let $B$ be an inverse-strongly monotone mapping of $C$ into $H$ and let $N_{C} v$ be normal cone to $C$ at $v \in C$, i.e., $N_{C} v=\{w \in H:\langle v-u, w\rangle \geq 0, \forall u \in C\}$, and define

$$
M v= \begin{cases}B v+N_{C} v, & \text { if } v \in C, \\ \varnothing, & \text { if } v \notin C .\end{cases}
$$

Then $M$ is a maximal monotone and $\theta \in M v$ if and only if $v \in V I(C, B)$ [16].

Let $M: H \rightarrow 2^{H}$ be a set-valued maximal monotone mapping, then the single-valued mapping $J_{M, \lambda}: H \rightarrow H$ defined by

$$
J_{M, \lambda}(x)=(I+\lambda M)^{-1}(x), \quad x \in H
$$

is called the resolvent operator associated with $M$, where $\lambda$ is any positive number and $I$ is the identity mapping. In the worth mentioning that the resolvent operator is nonexpansive, 1 -inverse-strongly monotone and that a solution of problem (1.8) is a fixed point of the operator $J_{M, \lambda}(I-\lambda B)$ for all $\lambda>0$ (see also [17]).

In 2000, Moudafi [18] introduced the viscosity approximation method for nonexpansive mapping and proved that if $H$ is a real Hilbert space, the sequence $\left\{x_{n}\right\}$ defined by the iterative method below, with the initial guess $x_{0} \in C$ is chosen arbitrarily,

$$
x_{n+1}=\alpha_{n} f\left(x_{n}\right)+\left(1-\alpha_{n}\right) S x_{n}, \quad n \geq 0,
$$

where $\left\{\alpha_{n}\right\} \subset(0,1)$ satisfies certain conditions, converge strongly to a fixed point of $S$ (say $\bar{x} \in C$ ) which is the unique solution of the following variational inequality. 
In 2005, Iiduka and Takahashi [19] introduced following iterative process $x_{0} \in C$,

$$
x_{n+1}=\alpha_{n} u+\left(1-\alpha_{n}\right) S P_{C}\left(x_{n}-\lambda_{n} A x_{n}\right), \quad \forall n \geq 0,
$$

where $u \in C,\left\{\alpha_{n}\right\} \subset(0,1)$ and $\left\{\lambda_{n}\right\} \subset[a, b]$ for some $a, b$ with $0<a<b<2 \beta$. They proved that under certain appropriate conditions imposed on $\left\{\alpha_{n}\right\}$ and $\left\{\lambda_{n}\right\}$, the sequence $\left\{x_{n}\right\}$ converges strongly to a common element of the set of fixed points of nonexpansive mapping and the set of solutions of the variational inequality for an inverse-strongly monotone mapping $($ say $\bar{x} \in C$ ) which solve some variational inequality.

In 2006, Marino and Xu [8] introduced a general iterative method for nonexpansive mapping. They defined the sequence $\left\{x_{n}\right\}$ generated by the algorithm $x_{0} \in C$,

$$
x_{n+1}=\alpha_{n} \gamma f\left(x_{n}\right)+\left(I-\alpha_{n} A\right) S x_{n}, \quad n \geq 0
$$

where $\left\{\alpha_{n}\right\} \subset(0,1)$ and $A$ is a strongly positive linear bounded operator. They proved that if $C=H$ then the sequence $\left\{x_{n}\right\}$ converges strongly to a fixed point of $S$ (say $\bar{x} \in H$ ) which is the unique solution of the following variational inequality.

In 2008, Su et al. [20] introduced the following iterative scheme by the viscosity approximation method in a real Hilbert space: $x_{1}, u_{n} \in H$

$$
\left\{\begin{array}{l}
F\left(u_{n}, y\right)+\frac{1}{r_{n}}\left\langle y-u_{n}, u_{n}-x_{n}\right\rangle \geq 0, \quad \forall y \in C \\
x_{n+1}=\alpha_{n} f\left(x_{n}\right)+\left(1-\alpha_{n}\right) S P_{C}\left(u_{n}-\lambda_{n} A u_{n}\right)
\end{array}\right.
$$

for all $n \in \mathbb{N}$, where $\left\{\alpha_{n}\right\} \subset[0,1)$ and $\left\{r_{n}\right\} \subset(0, \infty)$ satisfy some appropriate conditions. Furthermore, they proved $\left\{x_{n}\right\}$ and $\left\{u_{n}\right\}$ converge strongly to the same point $z$, where $z=P_{F(S) \cap V I(C, A) \cap E \mathrm{P}(F)} f(z)$.

In 2011, Tan and Chang [14] introduced following iterative process for $\left\{T_{n}: C \rightarrow C\right\}$ be a sequence of nonexpansive mappings. Let $\left\{x_{n}\right\}$ be the sequence defined by

$$
x_{n+1}=\alpha_{n} x_{n}+\left(1-\alpha_{n}\right)\left(S P_{C}\left(\left(1-t_{n}\right) J_{M, \lambda}(I-\lambda A) T_{n}(I-\mu B)\right) x_{n}\right), \quad \forall n \geq 0,
$$

where $\left\{\alpha_{n}\right\} \subset(0,1), \lambda \in(0,2 \alpha]$ and $\mu \in(0,2 \beta]$. The sequence $\left\{x_{n}\right\}$ converges strongly to a common element of the set of fixed points of nonexpansive mapping, the set of solutions of the variational inequality and the generalized equilibrium problem.

In this article, we modify by Marino and Xu [8], Su et al. [20] and Tan and Chang [14], the purpose of this article, we show that under some control conditions the sequence $\left\{x_{n}\right\}$ converges strongly to a common element of the set of fixed points of nonexpansive mappings, the solution of the generalized mixed equilibrium problems and the solution of the variational inclusions in a real Hilbert space.

\section{Preliminaries}

Let $H$ be a real Hilbert space and $C$ be a nonempty closed convex subset of $H$. We denote weak convergence and strong convergence by notations $\rightarrow$ and $\rightarrow$, respectively. Recall that the metric (nearest point) projection $P_{C}$ from $H$ onto $C$ assigns to each $x \in$ $H$, the unique point in $P_{C} x \in C$ satisfying the property

$$
\left\|x-P_{C} x\right\|=\inf _{y \in C}\|x-y\| .
$$


The following characterizes the projection $P_{C}$. We recall some lemmas which will be needed in the rest of this article.

Lemma 2.1. The function $u \in C$ is a solution of the variational inequality (1.5) if and only if $u \in C$ satisfies the relation $u=P_{C}(u-\lambda \Psi u)$ for all $\lambda>0$.

Lemma 2.2. For a given $z \in H, u \in C, u=P_{C} z \Leftrightarrow\langle u-z, v-u\rangle \geq 0, \forall v \in C$.

It is well known that $P_{C}$ is a firmly nonexpansive mapping of $H$ onto $C$ and satisfies

$$
\left\|P_{C} x-P_{C} y\right\|^{2} \leq\left\langle P_{C} x-P_{C} y, x-y\right\rangle, \quad \forall x, y \in H .
$$

Moreover, $P_{C} x$ is characterized by the following properties: $P_{C} x \in C$ and for all $x \in H$, $y \in C$

$$
\left\langle x-P_{C} x, y-P_{C} x\right\rangle \leq 0 .
$$

Lemma 2.3. [21] Let $M: H \rightarrow 2^{H}$ be a maximal monotone mapping and let $B: H \rightarrow H$ be a monotone and Lipshitz continuous mapping. Then the mapping $L=M+B: H \rightarrow 2^{H}$ is a maximal monotone mapping.

Lemma 2.4. [22]Each Hilbert space H satisfies Opial's condition, that is, for any sequence $\left\{x_{n}\right\} \subset H$ with $x_{n} \rightarrow x$, the inequality $\lim \inf _{n \rightarrow \infty}\left\|x_{n}-x\right\|<\lim _{\inf _{n \rightarrow \infty}}|| x_{n}-y \|$, hold for each $y \in H$ with $y \neq x$.

Lemma 2.5. [23]Assume $\left\{a_{n}\right\}$ is a sequence of nonnegative real numbers such that

$$
a_{n+1} \leq\left(1-\gamma_{n}\right) a_{n}+\delta_{n}, \quad \forall n \geq 0,
$$

where $\left\{\gamma_{n}\right\} \subset(0,1)$ and $\left\{\delta_{n}\right\}$ is a sequence in $\mathcal{R}$ such that

(i) $\sum_{n=1}^{\infty} \gamma_{n}=\infty$.

(ii) $\lim \sup _{n \rightarrow \infty} \frac{\delta_{n}}{\gamma_{n}} \leq 0$ or $\sum_{n=1}^{\infty}\left|\delta_{n}\right|<\infty$.

Then $\lim _{n \rightarrow \infty} a_{n}=0$.

Lemma 2.6. [24] Let $C$ be a closed convex subset of a real Hilbert space $H$ and let $S$ : $C \rightarrow C$ be a nonexpansive mapping. Then $I-S$ is demiclosed at zero, that is,

$$
x_{n} \rightarrow x, x_{n}-S x_{n} \rightarrow 0
$$

implies $x=S x$.

For solving the mixed equilibrium problem, let us assume that the bifunction $F: C \times C \rightarrow \mathcal{R}$ and $\varphi: C \rightarrow \mathcal{R}$ satisfies the following conditions:

(A1) $F(x, x)=0$ for all $x \in C$;

(A2) $F$ is monotone, i.e., $F(x, y)+F(y, x) \leq 0$ for any $x, y \in C$;

(A3) for each fixed $y \in C, x \mapsto F(x, y)$ is weakly upper semicontinuous;

(A4) for each fixed $x \in C, y \mapsto F(x, y)$ is convex and lower semicontinuous;

(B1) for each $x \in C$ and $r>0$, there exist a bounded subset $D_{x} \subseteq C$ and $y_{x} \in C$ such that for any $z \in C \backslash D_{x}$,

$$
F\left(z, \gamma_{x}\right)+\varphi\left(y_{x}\right)-\varphi(z)+\frac{1}{r}\left\langle y_{x}-z, z-x\right\rangle<0,
$$

(B2) $C$ is a bounded set. 
Lemma 2.7. [25] Let $C$ be a nonempty closed convex subset of a real Hilbert space $H$. Let $F: C \times C \rightarrow \mathcal{R}$ be a bifunction mapping satisfies (A1)-(A4) and let $\varphi: C \rightarrow \mathcal{R}$ is convex and lower semicontinuous such that $C \cap \operatorname{dom} \varphi \neq \emptyset$. Assume that either (B1) or (B2) holds. For $r>0$ and $x \in H$, then there exists $z \in C$ such that

$$
F(z, y)+\varphi(y)-\varphi(z)+\frac{1}{r}\langle y-z, z-x\rangle \geq 0, \quad \forall y \in C .
$$

Define a mapping $T_{r}^{(F, \varphi)}: H \rightarrow C$ as follows:

$$
T_{r}^{(F, \varphi)}(x)=\left\{z \in C: F(z, y)+\varphi(y)-\varphi(z)+\frac{1}{r}\langle y-z, z-x\rangle \geq 0, \quad \forall y \in C\right\}
$$

for all $x \in H$. Then, the following hold:

(i) $T_{r}^{(F, \varphi)}$ is single-valued;

(ii) $T_{r}^{(F, \varphi)}$ is firmly nonexpansive, i.e., for any $x, y \in H$,

$$
\left\|T_{r}^{(F, \varphi)} x-T_{r}^{(F, \varphi)} \gamma\right\|^{2} \leq\left\langle T_{r}^{(F, \varphi)} x-T_{r}^{(F, \varphi)} y, x-\gamma\right\rangle ;
$$

(iii) $F\left(T_{r}^{(F, \varphi)}\right)=\operatorname{MEP}(F, \varphi)$;

(iv) $\operatorname{MEP}(F, \phi)$ is closed and convex.

Lemma 2.8. [8]Assume $A$ is a strongly positive linear bounded operator on a Hilbert space $H$ with coefficient $\bar{\gamma}>0$ and $0<\rho \leq\|A\|^{-1}$, then $\|I-\rho A\| \leq 1-\rho \bar{\gamma}$.

Lemma 2.9. [26] Let $H$ be a real Hilbert space and $A: H \rightarrow H$ a mapping.

(i) If $A$ is a $\delta$-strongly monotone and $\mu$-strictly pseudo-contraction with $\delta+\mu>1$, then $I-A$ is a contraction with constant $\sqrt{(1-\delta) / \mu}$.

(ii) If $A$ is a $\delta$-strongly monotone and $\mu$-strictly pseudo-contraction with $\delta+\mu>1$, then for any fixed number $\tau \in(0,1), I-\tau A$ is a contraction with constant $1-\tau(1-\sqrt{(1-\delta) / \mu})$.

\section{Strong convergence theorems}

In this section, we show a strong convergence theorem which solves the problem of finding a common element of $F(S), \operatorname{GMEP}\left(F_{1}, \phi_{1}, B_{1}\right), \operatorname{GMEP}\left(F_{2}, \phi_{2}, B_{2}\right), I\left(A_{1}, M_{1}\right)$ and $I\left(A_{2}, M_{2}\right)$.

Theorem 3.1. Let $H$ be a real Hilbert space, $C$ be a closed convex subset of $H$. Let $F_{1}$, $F_{2}$ be bifunctions of $C \times C$ into $\mathcal{R}$ satisfying (A1)-(A4) and $A_{1}, A_{2}, B_{1}, B_{2}: C \rightarrow H$ be $\beta_{1}, \beta_{2}, \eta$, $\rho$-inverse-strongly monotone mappings, $\varphi_{1}, \varphi_{2}: C \rightarrow \mathcal{R}$ be convex and lower semicontinuous functions, $f: C \rightarrow C$ be a contraction with coefficient $\alpha(0<\alpha<1), M_{1}$, $M_{2}: H \rightarrow 2^{H}$ be maximal monotone mappings and $A$ is a $\delta$-strongly monotone and $\mu$ strictly pseudo-contraction with $\delta+\mu>1, \gamma$ is a positive real number such that 
$\gamma<\frac{1}{\alpha}\left(1-\sqrt{\frac{1-\delta}{\mu}}\right)$. Assume that either (B1) or (B2) holds. Let $S$ be a nonexpansive mapping of $C$ into itself such that

$$
\Theta:=F(S) \cap \operatorname{GMEP}\left(F_{1}, \varphi_{1}, B_{1}\right) \cap \operatorname{GMEP}\left(F_{2}, \varphi_{2}, B_{2}\right) \cap I\left(A_{1}, M_{1}\right) \cap I\left(A_{2}, M_{2}\right) \neq \emptyset .
$$

Suppose $\left\{x_{n}\right\}$ is a sequences generated by the following algorithm $x_{0} \in C$ arbitrarily:

$$
\left\{\begin{array}{l}
u_{n}=T_{r_{n}}^{\left(F_{1}, \varphi_{1}\right)}\left(x_{n}-r_{n} B_{1} x_{n}\right) \\
v_{n}=T_{\left.s_{n}, \varphi_{2}\right)}^{\left(F_{n}\right.}\left(x_{n}-s_{n} B_{2} x_{n}\right) \\
x_{n+1}=\xi_{n} P_{C}\left\{\alpha_{n} \gamma f\left(x_{n}\right)+\left(I-\alpha_{n} A\right) S P_{C}\left[J_{M_{1}, \lambda_{1}}\left(1-\lambda_{1} A_{1}\right) u_{n}\right]\right\} \\
+\left(1-\xi_{n}\right) P_{C}\left[J_{M_{2}, \lambda_{2}}\left(I-\lambda_{2} A_{2}\right) v_{n}\right], \quad \forall n \geq 0,
\end{array}\right.
$$

where $\left\{\alpha_{n}\right\},\left\{\xi_{n}\right\} \subset(0,1), \lambda_{1} \in\left(0,2 \beta_{1}\right)$ such that $0<a_{1} \leq \lambda_{1}<b_{1}<2 \beta_{1}, \lambda_{2} \in\left(0,2 \beta_{2}\right)$ such that $0<a_{1} \leq \lambda_{2} \leq b_{2}<2 \beta_{2}, r_{n} \in(0,2 \eta)$ with $0<c \leq d \leq 1-\eta$ and $s_{n} \in(0,2 \rho)$ with $0<e \leq f \leq 1-\rho$ satisfy the following conditions:

(C1): $\lim _{n \rightarrow \infty} \alpha_{n}=0, \sum_{n=0}^{\infty} \alpha_{n}=\infty, \sum_{n=1}^{\infty}\left|\alpha_{n+1}-\alpha_{n}\right|<\infty$,

(C2): $0<\lim \inf _{n \rightarrow \infty} \xi_{n}<\lim \sup _{n \rightarrow \infty} \xi_{n}<1, \sum_{n=1}^{\infty}\left|\xi_{n+1}-\xi_{n}\right|<\infty$,

(C3): $\lim _{\inf _{n \rightarrow \infty}} r_{n}>0$ and $\lim _{n \rightarrow \infty}\left|r_{n+1}-r_{n}\right|=0$,

(C4): $\lim _{\inf _{n \rightarrow \infty}} s_{n}>0$ and $\lim _{n \rightarrow \infty}\left|s_{n+1}-s_{n}\right|=0$.

Then $\left\{x_{n}\right\}$ converges strongly to $q \in \Theta$, where $q=P_{\Theta}(\gamma f+I-A)(q)$ which solves the following variational inequality:

$$
\langle(\gamma f-A) q, p-q\rangle \leq 0, \quad \forall p \in \Theta
$$

which is the optimality condition for the minimization problem

$$
\min _{q \in \Theta} \frac{1}{2}\langle A q, q\rangle-h(q)
$$

where $h$ is a potential function for $\gamma f\left(\right.$ i.e., $h^{\prime}(q)=\gamma f(q)$ for $\left.q \in H\right)$.

Proof. Since $A_{1}, A_{2}$ are $\beta_{1}, \beta_{2}$-inverse-strongly monotone mappings, we have

$$
\begin{aligned}
\left\|\left(I-\lambda_{1} A_{1}\right) x-\left(I-\lambda_{1} A_{1}\right) y\right\|^{2} & =\left\|(x-y)-\lambda_{1}\left(A_{1} x-A_{1} \gamma\right)\right\|^{2} \\
& =\|x-y\|^{2}-2 \lambda_{1}\left\langle x-\gamma, A_{1} x-A_{1} \gamma\right\rangle+\lambda_{1}^{2}\left\|A_{1} x-A_{1} \gamma\right\|^{2} \\
& \leq\|x-y\|^{2}+\lambda_{1}\left(\lambda_{1}-2 \beta_{1}\right)\left\|A_{1} x-A_{1} \gamma\right\|^{2} \\
& \leq\|x-y\|^{2} .
\end{aligned}
$$

In similar way, we can obtain

$$
\left\|\left(I-\lambda_{2} A_{2}\right) x-\left(I-\lambda_{2} A_{2}\right) y\right\|^{2} \leq\|x-y\|^{2} .
$$


And $B_{1}, B_{2}$ are $\eta, \rho$-inverse-strongly monotone mappings, we have

$$
\begin{aligned}
\left\|\left(I-r_{n} B_{1}\right) x-\left(I-r_{n} B_{1}\right) y\right\|^{2} & =\left\|(x-y)-r_{n}\left(B_{1} x-B_{1} y\right)\right\|^{2} \\
& =\|x-\gamma\|^{2}-2 r_{n}\left\langle x-\gamma, B_{1} x-B_{1} \gamma\right\rangle+r_{n}^{2}\left\|B_{1} x-B_{1} y\right\|^{2} \\
& \leq\|x-\gamma\|^{2}+r_{n}\left(r_{n}-2 \eta\right)\left\|B_{1} x-B_{1} y\right\|^{2} \\
& \leq\|x-\gamma\|^{2} .
\end{aligned}
$$

In similar way, we can obtain

$$
\left\|\left(I-s_{n} B_{2}\right) x-\left(I-s_{n} B_{2}\right) y\right\|^{2} \leq\|x-y\|^{2} .
$$

It is clear that if $0<\lambda_{1}<2 \beta_{1}, 0<\lambda_{2}<2 \beta_{2}, 0<r_{n}<2 \eta, 0<s_{n} \leq 2 \rho$ then $I-\lambda_{1} A_{1}, I-$ $\lambda_{2} A_{2}, I-r_{n} B_{1}, I-s_{n} B_{2}$ are all nonexpansive. We will divide the proof into six steps.

Step 1. We will show $\left\{x_{n}\right\}$ is bounded. Put $y_{n}=J_{M_{1}, \lambda_{1}}\left(u_{n}-\lambda_{1} A_{1} u_{n}\right)$ for all $n \geq 0$ and $w_{n}=J_{M_{2}, \lambda_{2}}\left(v_{n}-\lambda_{2} A_{2} v_{n}\right)$ for all $n \geq 0$. It follows that

$$
\begin{aligned}
\left\|y_{n}-q\right\| & =\left\|J_{M_{1}, \lambda_{1}}\left(u_{n}-\lambda_{1} A_{1} u_{n}\right)-J_{M_{1}, \lambda_{1}}\left(q-\lambda_{1} A_{1} q\right)\right\| \\
& \leq\left\|u_{n}-q\right\| .
\end{aligned}
$$

In similar way, we can obtain

$$
\begin{aligned}
\left\|w_{n}-q\right\| & =\left\|J_{M_{2}, \lambda_{2}}\left(v_{n}-\lambda_{2} A_{2} v_{n}\right)-J_{M_{2}, \lambda_{2}}\left(q-\lambda_{2} A_{2} q\right)\right\| \\
& \leq\left\|v_{n}-q\right\| .
\end{aligned}
$$

Put $y_{n}^{\prime}=P_{C} y_{n}, n \geq 0$. It follows that

$$
\begin{aligned}
\left\|y_{n}^{\prime}-q\right\| & =\left\|P_{C} y_{n}-P_{C} q\right\| \\
& \leq\left\|y_{n}-q\right\| .
\end{aligned}
$$

By Lemma 2.7, we have $u_{n}=T_{r_{n}}^{\left(F_{1}, \varphi_{1}\right)}\left(x_{n}-r_{n} B_{1} x_{n}\right), v_{n}=T_{s_{n}}^{\left(F_{2}, \varphi_{2}\right)}\left(x_{n}-s_{n} B_{2} x_{n}\right)$ for all $n$ $\geq 0$. Then, we have

$$
\begin{aligned}
\left\|u_{n}-q\right\|^{2} & =\left\|T_{r_{n}}^{\left(F_{1}, \varphi_{1}\right)}\left(x_{n}-r_{n} B_{1} x_{n}\right)-T_{r_{n}}^{\left(F_{1}, \varphi_{1}\right)}\left(q-r_{n} B_{1} q\right)\right\|^{2} \\
& \leq\left\|\left(x_{n}-r_{n} B_{1} x_{n}\right)-\left(q-r_{n} B_{1} q\right)\right\|^{2} \\
& \leq\left\|x_{n}-q\right\|^{2}+r_{n}\left(r_{n}-2 \eta\right)\left\|B_{1} x_{n}-B_{1} q\right\|^{2} \\
& \leq\left\|x_{n}-q\right\|^{2} .
\end{aligned}
$$

In similar way, we can obtain

$$
\begin{aligned}
\left\|v_{n}-q\right\|^{2} & =\left\|T_{s_{n}}^{\left(F_{2}, \varphi_{2}\right)}\left(x_{n}-s_{n} B_{2} x_{n}\right)-T_{s_{n}}^{\left(F_{2}, \varphi_{2}\right)}\left(q-s_{n} B_{2} q\right)\right\|^{2} \\
& \leq\left\|\left(x_{n}-s_{n} B_{2} x_{n}\right)-\left(q-s_{n} B_{2} q\right)\right\|^{2} \\
& \leq\left\|x_{n}-q\right\|^{2}+s_{n}\left(s_{n}-2 \rho\right)\left\|B_{2} x_{n}-B_{2} q\right\|^{2} \\
& \leq\left\|x_{n}-q\right\|^{2} .
\end{aligned}
$$

Put $z_{n}=P_{C}\left[\alpha_{n} \gamma f\left(x_{n}\right)+\left(I-\alpha_{n} A\right) S P_{C} y_{n}\right]$ for all $n \geq 0$. From (3.1) and by Lemma 2.9 
(ii), we deduce that

$$
\begin{aligned}
\left\|x_{n+1}-q\right\|= & \left\|\xi_{n}\left(z_{n}-q\right)+\left(1-\xi_{n}\right)\left(P_{C} w_{n}-q\right)\right\| \\
\leq & \xi_{n}\left\|P_{C}\left[\alpha_{n} \gamma f\left(x_{n}\right)+\left(I-\alpha_{n} A\right) S P_{C} y_{n}\right]-P_{C} q\right\|+\left(1-\xi_{n}\right)\left\|P_{C} w_{n}-P_{C} q\right\| \\
\leq & \xi_{n}\left\|\alpha_{n} \gamma f\left(x_{n}\right)+\left(I-\alpha_{n} A\right) S P_{C} y_{n}-q\right\|+\left(1-\xi_{n}\right)\left\|w_{n}-q\right\| \\
= & \xi_{n}\left\|\alpha_{n}\left(\gamma f\left(x_{n}\right)-A q\right)+\left(I-\alpha_{n} A\right)\left(S P_{C} y_{n}-q\right)\right\|+\left(1-\xi_{n}\right)\left\|w_{n}-q\right\| \\
\leq & \xi_{n} \alpha_{n}\left\|\gamma f\left(x_{n}\right)-A q\right\| \\
& +\xi_{n}\left(1-\alpha_{n}\left(1-\sqrt{\frac{1-\delta}{\mu}}\right)\right)\left\|P_{C} y_{n}-q\right\|+\left(1-\xi_{n}\right)\left\|w_{n}-q\right\| \\
\leq & \xi_{n} \alpha_{n}\left\|\gamma f\left(x_{n}\right)-\gamma f(q)\right\|+\xi_{n} \alpha_{n}\|\gamma f(q)-A q\| \\
& +\xi_{n}\left(1-\alpha_{n}\left(1-\sqrt{\frac{1-\delta}{\mu}}\right)\right)\left\|P_{C} y_{n}-q\right\|+\left(1-\xi_{n}\right)\left\|x_{n}-q\right\| \\
\leq & \xi_{n} \alpha_{n} \gamma \alpha\left\|x_{n}-q\right\|+\xi_{n} \alpha_{n}\|\gamma f(q)-A q\| \\
& +\xi_{n}\left(1-\alpha_{n}\left(1-\sqrt{\frac{1-\delta}{\mu}}\right)\right)\left\|y_{n}-q\right\|+\left(1-\xi_{n}\right)\left\|x_{n}-q\right\| \\
= & \left(1-\left(\left(1-\sqrt{\frac{1-\delta}{\mu}}\right)-\gamma \alpha\right) \xi_{n} \alpha_{n}\right)\left\|x_{n}-q\right\|+\xi_{n} \alpha_{n}\|\gamma f(q)-A q\| \\
\leq & \left(1-\left(\left(1-\sqrt{\frac{1-\delta}{\mu}}\right)-\gamma \alpha\right) \xi_{n} \alpha_{n}\right)\left\|x_{n}-q\right\| \\
& +\left(\left(1-\sqrt{\frac{1-\delta}{\mu}}\right)-\gamma \alpha\right) \xi_{n} \alpha_{n} \frac{\|\gamma f(q)-A q\|}{\left(\left(1-\sqrt{\frac{1-\delta}{\mu}}\right)-\gamma \alpha\right)} \\
\leq & \max \left\{\left\|x_{n}-q\right\|, \frac{\|\gamma f(q)-A q\|}{\left(1-\sqrt{\frac{1-\delta}{\mu}}\right)-\gamma \alpha}\right\} . \\
& (10.6)
\end{aligned}
$$

It follows from induction that

$$
\left\|x_{n}-q\right\| \leq \max \left\{\left\|x_{0}-q\right\|, \frac{\|\gamma f(q)-A q\|}{\left(1-\sqrt{\frac{1-\delta}{\mu}}\right)-\gamma \alpha}\right\}, \quad n \geq 0 .
$$

Therefore $\left\{x_{n}\right\}$ is bounded, so are $\left\{y_{n}\right\},\left\{z_{n}\right\},\left\{P_{C} w_{n}\right\},\left\{S P_{C} y_{n}\right\},\left\{f\left(x_{n}\right)\right\}$ and $\left\{A S P_{C} y_{n}\right\}$.

Step 2. We claim that $\lim _{n \rightarrow \infty}|| x_{n+2}-x_{n+1} \|=0$. From (3.1), we have

$$
\begin{aligned}
\left\|x_{n+2}-x_{n+1}\right\|= & \left\|\xi_{n+1} z_{n+1}+\left(1-\xi_{n+1}\right) P_{C} w_{n+1}-\xi_{n} z_{n}-\left(1-\xi_{n}\right) P_{C} w_{n}\right\| \\
= & \| \xi_{n+1}\left(z_{n+1}-z_{n}\right)+\left(\xi_{n+1}-\xi_{n}\right) z_{n} \\
& +\left(1-\xi_{n+1}\right)\left(P_{C} w_{n+1}-P_{C} w_{n}\right)+\left(\xi_{n}-\xi_{n+1}\right) P_{C} w_{n} \| \\
\leq & \xi_{n+1}\left\|z_{n+1}-z_{n}\right\|+\left(1-\xi_{n+1}\right)\left\|w_{n+1}-w_{n}\right\| \\
& +\left|\xi_{n+1}-\xi_{n}\right|\left(\left\|z_{n}\right\|+\left\|P_{C} w_{n}\right\|\right) .
\end{aligned}
$$


Since $I-\lambda_{2} A_{2}$ be nonexpansive, we have

$$
\begin{aligned}
\left\|w_{n+1}-w_{n}\right\| & =\left\|J_{M_{2}, \lambda_{2}}\left(v_{n+1}-\lambda_{2} A_{2} v_{n+1}\right)-J_{M_{2}, \lambda_{2}}\left(v_{n}-\lambda_{2} A_{2} v_{n}\right)\right\| \\
& \leq\left\|\left(v_{n+1}-\lambda_{2} A_{2} v_{n+1}\right)-\left(v_{n}-\lambda_{2} A_{2} v_{n}\right)\right\| \\
& \leq\left\|v_{n+1}-v_{n}\right\| .
\end{aligned}
$$

On the other hand, from $v_{n-1}=T_{s_{n-1}}^{\left(F_{2}, \varphi_{2}\right)}\left(x_{n-1}-s_{n-1} B_{2} x_{n-1}\right)$ and $v_{n}=T_{s_{n}}^{\left(F_{2}, \varphi_{2}\right)}\left(x_{n}-s_{n} B_{2} x_{n}\right)$, it follows that

$$
\begin{gathered}
F_{2}\left(v_{n-1}, y\right)+\left\langle B_{2} x_{n-1}, y-v_{n-1}\right\rangle+\varphi_{2}(y)-\varphi_{2}\left(v_{n-1}\right) \\
+\frac{1}{s_{n-1}}\left\langle y-v_{n-1}, v_{n-1}-x_{n-1}\right\rangle \geq 0, \quad \forall y \in C
\end{gathered}
$$

and

$$
F_{2}\left(v_{n}, y\right)+\left\langle B_{2} x_{n}, y-v_{n}\right\rangle+\varphi_{2}(y)-\varphi_{2}\left(v_{n}\right)+\frac{1}{s_{n}}\left\langle y-v_{n}, v_{n}-x_{n}\right\rangle \geq 0, \quad \forall y \in C(3.12)
$$

Substituting $y=v_{n}$ in (3.11) and $y=v_{n-1}$ in (3.12), we get

$$
F_{2}\left(v_{n-1}, v_{n}\right)+\left\langle B_{2} x_{n-1}, v_{n}-v_{n-1}\right\rangle+\varphi_{2}\left(v_{n}\right)-\varphi_{2}\left(v_{n-1}\right)+\frac{1}{s_{n-1}}\left\langle v_{n}-v_{n-1}, v_{n-1}-x_{n-1}\right\rangle \geq 0
$$

and

$$
F_{2}\left(v_{n}, v_{n-1}\right)+\left\langle B_{2} x_{n}, v_{n-1}-v_{n}\right\rangle+\varphi_{2}\left(v_{n-1}\right)-\varphi_{2}\left(v_{n}\right)+\frac{1}{s_{n}}\left\langle v_{n-1}-v_{n}, v_{n}-x_{n}\right\rangle \geq 0 .
$$

From (A2), we obtain

$$
\left\langle v_{n}-v_{n-1}, B_{2} x_{n-1}-B_{2} x_{n}+\frac{v_{n-1}-x_{n-1}}{s_{n-1}}-\frac{v_{n}-x_{n}}{s_{n}}\right\rangle \geq 0,
$$

and then

$$
\left\langle v_{n}-v_{n-1}, s_{n-1}\left(B_{2} x_{n-1}-B_{2} x_{n}\right)+v_{n-1}-x_{n-1}-\frac{s_{n-1}}{s_{n}}\left(v_{n}-x_{n}\right)\right\rangle \geq 0,
$$

so

$$
\left\langle v_{n}-v_{n-1}, s_{n-1} B_{2} x_{n-1}-s_{n-1} B_{2} x_{n}+v_{n-1}-v_{n}+v_{n}-x_{n-1}-\frac{s_{n-1}}{s_{n}}\left(v_{n}-x_{n}\right)\right\rangle \geq 0 .
$$

It follows that

$$
\begin{gathered}
\left\langle v_{n}-v_{n-1},\left(I-s_{n-1} B_{2}\right) x_{n}-\left(I-s_{n-1} B_{2}\right) x_{n-1}\right. \\
\left.+v_{n-1}-v_{n}+v_{n}-x_{n}-\frac{s_{n-1}}{s_{n}}\left(v_{n}-x_{n}\right)\right\rangle \geq 0 \\
\left\langle v_{n}-v_{n-1}, v_{n-1}-v_{n}\right\rangle+\left\langle v_{n}-v_{n-1}, x_{n}-x_{n-1}+\left(1-\frac{s_{n-1}}{s_{n}}\right)\left(v_{n}-x_{n}\right)\right\rangle \geq 0 .
\end{gathered}
$$

Without loss of generality, let us assume that there exists a real number $e$ such that $s_{n-1}>e>0$, for all $n \in \mathbb{N}$. Then, we have 


$$
\begin{aligned}
\left\|v_{n}-v_{n-1}\right\|^{2} & \leq\left\langle v_{n}-v_{n-1}, x_{n}-x_{n-1}+\left(1-\frac{s_{n-1}}{s_{n}}\right)\left(v_{n}-x_{n}\right)\right\rangle \\
& \leq\left\|v_{n}-v_{n-1}\right\|\left\{\left\|x_{n}-x_{n-1}\right\|+\left|1-\frac{s_{n-1}}{s_{n}}\right|\left\|v_{n}-x_{n}\right\|\right\}
\end{aligned}
$$

and hence

$$
\begin{aligned}
\left\|v_{n}-v_{n-1}\right\| & \leq\left\|x_{n}-x_{n-1}\right\|+\frac{1}{s_{n}}\left|s_{n}-s_{n-1}\right|\left\|v_{n}-x_{n}\right\| \\
& \leq\left\|x_{n}-x_{n-1}\right\|+\frac{M_{1}}{e}\left|s_{n}-s_{n-1}\right|
\end{aligned}
$$

where $M_{1}=\sup \left\{\left\|v_{n}-x_{n}\right\|: n \in \mathbb{N}\right\}$. Substituting (3.13) into (3.9) and (3.10) that

$$
\begin{aligned}
\left\|x_{n+2}-x_{n+1}\right\| \leq & \xi_{n+1}\left\|z_{n+1}-z_{n}\right\|+\left(1-\xi_{n+1}\right)\left\{\left\|x_{n+1}-x_{n}\right\|+\frac{M_{1}}{e}\left|s_{n}-s_{n-1}\right|\right\} \\
& +\left|\xi_{n+1}-\xi_{n}\right|\left(\left\|z_{n}\right\|+\left\|P_{C} w_{n}\right\|\right) .
\end{aligned}
$$

By Lemma 2.9 (ii), it follow that

$$
\begin{aligned}
&\left\|z_{n+1}-z_{n}\right\| \\
&= \| P_{C}\left[\alpha_{n+1} \gamma f\left(x_{n+1}\right)+\left(I-\alpha_{n+1} A\right) S P_{C} y_{n+1}\right] \\
&-P_{C}\left[\alpha_{n} \gamma f\left(x_{n}\right)+\left(I-\alpha_{n} A\right) S P_{C} y_{n}\right] \| \\
& \leq\left\|\alpha_{n+1} \gamma f\left(x_{n+1}\right)+\left(I-\alpha_{n+1} A\right) S P_{C} y_{n+1}-\alpha_{n} \gamma f\left(x_{n}\right)-\left(I-\alpha_{n} A\right) S P_{C} y_{n}\right\| \\
&= \| \alpha_{n+1} \gamma\left(f\left(x_{n+1}\right)-f\left(x_{n}\right)\right)+\left(\alpha_{n+1}-\alpha_{n}\right) \gamma f\left(x_{n}\right) \\
&+\left(I-\alpha_{n+1} A\right)\left(S P_{C} y_{n+1}-S P_{C} y_{n}\right)+\left(\alpha_{n}-\alpha_{n+1}\right) A S P_{C} y_{n} \| \\
& \leq \alpha_{n+1} \gamma \alpha\left\|x_{n+1}-x_{n}\right\|+\left|\alpha_{n+1}-\alpha_{n}\right|\left\|\gamma f\left(x_{n}\right)\right\| \\
&+\left(1-\alpha_{n+1}\left(1-\sqrt{\frac{1-\delta}{\mu}}\right)\right)\left\|y_{n+1}-y_{n}\right\|+\left|\alpha_{n+1}-\alpha_{n}\right|\left\|A S P_{C} y_{n}\right\| \\
&= \alpha_{n+1} \gamma \alpha\left\|x_{n+1}-x_{n}\right\|+\left|\alpha_{n+1}-\alpha_{n}\right|\left(\left\|\gamma f\left(x_{n}\right)\right\|+\left\|A S P_{C} y_{n}\right\|\right) \\
&+\left(1-\alpha_{n+1}\left(1-\sqrt{\frac{1-\delta}{\mu}}\right)\right)\left\|y_{n+1}-y_{n}\right\| .
\end{aligned}
$$

Since $I-\lambda_{1} A_{1}$ be nonexpansive, we have

$$
\begin{aligned}
\left\|y_{n+1}-y_{n}\right\| & =\left\|J_{M_{1}, \lambda_{1}}\left(u_{n+1}-\lambda_{1} A_{1} u_{n+1}\right)-J_{M_{1}, \lambda_{1}}\left(u_{n}-\lambda_{1} A_{1} u_{n}\right)\right\| \\
& \leq\left\|\left(u_{n+1}-\lambda_{1} A_{1} u_{n+1}\right)-\left(u_{n}-\lambda_{1} A_{1} u_{n}\right)\right\| \\
& \leq\left\|\left(I-\lambda_{1} A_{1}\right) u_{n+1}-\left(I-\lambda_{1} A_{1}\right) u_{n}\right\| \\
& \leq\left\|u_{n+1}-u_{n}\right\| .
\end{aligned}
$$

On the other hand, from $u_{n-1}=T_{r_{n-1}}^{\left(F_{1}, \varphi_{1}\right)}\left(x_{n-1}-r_{n-1} B_{1} x_{n-1}\right)$ and $u_{n}=T_{r_{n}}^{\left(F_{1}, \varphi_{1}\right)}\left(x_{n}-r_{n} B_{1} x_{n}\right)$, it follows that

$$
\begin{gathered}
F_{1}\left(u_{n-1}, y\right)+\left\langle B_{1} x_{n-1}, y-u_{n-1}\right\rangle+\varphi_{1}(y)-\varphi_{1}\left(u_{n-1}\right) \\
+\frac{1}{r_{n-1}}\left\langle y-u_{n-1}, u_{n-1}-x_{n-1}\right\rangle \geq 0, \quad \forall y \in C
\end{gathered}
$$


and

$$
F_{1}\left(u_{n}, y\right)+\left\langle B_{1} x_{n}, y-u_{n}\right\rangle+\varphi_{1}(y)-\varphi_{1}\left(u_{n}\right)+\frac{1}{r_{n}}\left\langle y-u_{n}, u_{n}-x_{n}\right\rangle \geq 0, \quad \forall y \in C
$$

Substituting $y=u_{n}$ in (3.17) and $y=u_{n-1}$ in (3.18), we get

$$
F_{1}\left(u_{n-1}, u_{n}\right)+\left\langle B_{1} x_{n-1}, u_{n}-u_{n-1}\right\rangle+\varphi_{1}\left(u_{n}\right)-\varphi_{1}\left(u_{n-1}\right)+\frac{1}{r_{n-1}}\left\langle u_{n}-u_{n-1}, u_{n-1}-x_{n-1}\right\rangle \geq 0
$$

and

$$
F_{1}\left(u_{n}, u_{n-1}\right)+\left\langle B_{1} x_{n}, u_{n-1}-u_{n}\right\rangle+\varphi_{1}\left(u_{n-1}\right)-\varphi_{1}\left(u_{n}\right)+\frac{1}{r_{n}}\left\langle u_{n-1}-u_{n}, u_{n}-x_{n}\right\rangle \geq 0 .
$$

From (A2), we obtain

$$
\left\langle u_{n}-u_{n-1}, B_{1} x_{n-1}-B_{1} x_{n}+\frac{u_{n-1}-x_{n-1}}{r_{n-1}}-\frac{u_{n}-x_{n}}{r_{n}}\right\rangle \geq 0
$$

and then

$$
\left\langle u_{n}-u_{n-1}, r_{n-1}\left(B_{1} x_{n-1}-B_{1} x_{n}\right)+u_{n-1}-x_{n-1}-\frac{r_{n-1}}{r_{n}}\left(u_{n}-x_{n}\right)\right\rangle \geq 0,
$$

so

$$
\left\langle u_{n}-u_{n-1}, r_{n-1} B_{1} x_{n-1}-r_{n-1} B_{1} x_{n}+u_{n-1}-u_{n}+u_{n}-x_{n-1}-\frac{r_{n-1}}{r_{n}}\left(u_{n}-x_{n}\right)\right\rangle \geq 0 .
$$

It follows that

$$
\begin{gathered}
\left\langle u_{n}-u_{n-1},\left(I-r_{n-1} B_{1}\right) x_{n}-\left(I-r_{n-1} B_{1}\right) x_{n-1}\right. \\
\left.+u_{n-1}-u_{n}+u_{n}-x_{n}-\frac{r_{n-1}}{r_{n}}\left(u_{n}-x_{n}\right)\right\rangle \geq 0 \\
\left\langle u_{n}-u_{n-1}, u_{n-1}-u_{n}\right\rangle+\left\langle u_{n}-u_{n-1}, x_{n}-x_{n-1}+\left(1-\frac{r_{n-1}}{r_{n}}\right)\left(u_{n}-x_{n}\right)\right\rangle \geq 0 .
\end{gathered}
$$

Without loss of generality, let us assume that there exists a real number $c$ such that $r_{n-1}>c>0$, for all $n \in \mathbb{N}$. Then, we have

$$
\begin{aligned}
\left\|u_{n}-u_{n-1}\right\|^{2} & \leq\left\langle u_{n}-u_{n-1}, x_{n}-x_{n-1}+\left(1-\frac{r_{n-1}}{r_{n}}\right)\left(u_{n}-x_{n}\right)\right\rangle \\
& \leq\left\|u_{n}-u_{n-1}\right\|\left\{\left\|x_{n}-x_{n-1}\right\|+\left|1-\frac{r_{n-1}}{r_{n}}\right|\left\|u_{n}-x_{n}\right\|\right\}
\end{aligned}
$$

and hence

$$
\begin{aligned}
\left\|u_{n}-u_{n-1}\right\| & \leq\left\|x_{n}-x_{n-1}\right\|+\frac{1}{r_{n}}\left|r_{n}-r_{n-1}\right|\left\|u_{n}-x_{n}\right\| \\
& \leq\left\|x_{n}-x_{n-1}\right\|+\frac{M_{2}}{c}\left|r_{n}-r_{n-1}\right|,
\end{aligned}
$$


where $M_{2}=\sup \left\{\| u_{n}-x_{n}||: n \in \mathbb{N}\right\}$. Substituting (3.19) into (3.16), we have

$$
\left\|y_{n}-y_{n-1}\right\| \leq\left\|x_{n}-x_{n-1}\right\|+\frac{M_{2}}{c}\left|r_{n}-r_{n-1}\right| .
$$

Substituting (3.20) into (3.15), we obtain that

$$
\begin{aligned}
\left\|z_{n+1}-z_{n}\right\| \leq & \alpha_{n+1} \gamma \alpha\left\|x_{n+1}-x_{n}\right\|+\left|\alpha_{n+1}-\alpha_{n}\right|\left(\left\|\gamma f\left(x_{n}\right)\right\|+\left\|A S P_{C} y_{n}\right\|\right) \\
& +\left(1-\alpha_{n+1}\left(1-\sqrt{\frac{1-\delta}{\mu}}\right)\right)\left\{\left\|x_{n}-x_{n-1}\right\|+\frac{M_{2}}{c}\left|r_{n}-r_{n-1}\right|\right\}^{(3.21}
\end{aligned}
$$

And substituting (3.10), (3.13), (3.21) into (3.9), we get

$$
\begin{aligned}
\left\|x_{n+2}-x_{n+1}\right\| \leq & \xi_{n+1}\left\{\alpha_{n+1} \gamma \alpha\left\|x_{n+1}-x_{n}\right\|+\left|\alpha_{n+1}-\alpha_{n}\right|\left(\left\|\gamma f\left(x_{n}\right)\right\|+\left\|A S P_{C} y_{n}\right\|\right)\right. \\
& \left.+\left(1-\alpha_{n+1}\left(1-\sqrt{\frac{1-\delta}{\mu}}\right)\right)\left\|x_{n}-x_{n-1}\right\|+\frac{M_{2}}{c}\left|r_{n}-r_{n-1}\right|\right\} \\
& +\left(1-\xi_{n+1}\right)\left\{\left\|x_{n}-x_{n-1}\right\|+\frac{M_{1}}{e}\left|s_{n}-s_{n-1}\right|\right\} \\
& +\left|\xi_{n+1}-\xi_{n}\right|\left(\left\|z_{n}\right\|+\left\|P_{C} w_{n}\right\|\right) \\
\leq & \left(1-\left(\left(1-\sqrt{\frac{1-\delta}{\mu}}\right)-\gamma \alpha\right) \xi_{n+1} \alpha_{n+1}\right)\left\|x_{n+1}-x_{n}\right\| \\
& +\left(\left|\alpha_{n+1}-\alpha_{n}\right|+\left|\xi_{n+1}-\xi_{n}\right|\right) M_{3} \\
& +\frac{M_{2}}{c}\left|r_{n}-r_{n-1}\right|+\frac{M_{1}}{e}\left|s_{n}-s_{n-1}\right|
\end{aligned}
$$

where $M_{3}>0$ is a constant satisfying

$$
\sup _{n}\left\{\left\|\gamma f\left(x_{n}\right)\right\|+\left\|A S P_{C} y_{n}\right\|,\left\|z_{n}\right\|+\left\|P_{C} w_{n}\right\|\right\} \leq M_{3} \text {. }
$$

This together with (C1)-(C4) and Lemma 2.5, imply that

$$
\lim _{n \rightarrow \infty}\left\|x_{n+2}-x_{n+1}\right\|=0
$$

From (3.20) and (C3), we also have $\left\|y_{n+1}-y_{n}\right\| \rightarrow 0$ as $n \rightarrow \infty$.

Step 3. We show the followings:

(i) $\lim _{n \rightarrow \infty}\left\|A_{1} u_{n}-A_{1} q\right\|=0$;

(ii) $\lim _{n \rightarrow \infty}\left\|A_{2} v_{n}-A_{2} q\right\|=0$;

(iii) $\lim _{n \rightarrow \infty}\left\|B_{1} x_{n}-B_{1} q\right\|=0$;

(iv) $\lim _{n \rightarrow \infty}\left\|B_{2} x_{n}-B_{2} q\right\|=0$.

For $q \in \Theta$ and $q=J_{M_{1}, \lambda_{1}}\left(q-\lambda_{1} A_{1} q\right)$, then we get

$$
\begin{aligned}
\left\|y_{n}-q\right\|^{2} & =\left\|J_{M_{1}, \lambda_{1}}\left(u_{n}-\lambda_{1} A_{1} u_{n}\right)-J_{M_{1}, \lambda_{1}}\left(q-\lambda_{1} A_{1} q\right)\right\|^{2} \\
& \leq\left\|\left(u_{n}-\lambda_{1} A_{1} u_{n}\right)-\left(q-\lambda_{1} A_{1} q\right)\right\|^{2} \\
& \leq\left\|u_{n}-q\right\|^{2}+\lambda_{1}\left(\lambda_{1}-2 \beta_{1}\right)\left\|A_{1} u_{n}-A_{1} q\right\|^{2} \\
& \leq\left\|x_{n}-q\right\|^{2}+\lambda_{1}\left(\lambda_{1}-2 \beta_{1}\right)\left\|A_{1} u_{n}-A_{1} q\right\|^{2} .
\end{aligned}
$$


Using (3.5), it follows that

$$
\begin{aligned}
\left\|z_{n}-q\right\|^{2} & \left\|P_{C}\left[\alpha_{n} \gamma f\left(x_{n}\right)+\left(I-\alpha_{n} A\right) S \gamma_{n}^{\prime}\right]-P_{C}(q)\right\|^{2} \\
\leq & \left\|\alpha_{n}\left(\gamma f\left(x_{n}\right)-A q\right)+\left(I-\alpha_{n} A\right)\left(S \gamma_{n}^{\prime}-q\right)\right\|^{2} \\
\leq & \left\|\alpha_{n}\left(\gamma f\left(x_{n}\right)-A q\right)+\left(I-\alpha_{n} A\right)\left(\gamma_{n}^{\prime}-q\right)\right\|^{2} \\
\leq & \left\|\alpha_{n}\left(\gamma f\left(x_{n}\right)-A q\right)+\left(I-\alpha_{n} A\right)\left(\gamma_{n}-q\right)\right\|^{2} \\
\leq & \alpha_{n}\left\|\gamma f\left(x_{n}\right)-A q\right\|^{2}+\left(1-\alpha_{n}\left(1-\sqrt{\frac{1-\delta}{\mu}}\right)\right)\left\|y_{n}-q\right\|^{2} \\
& +2 \alpha_{n}\left(1-\alpha_{n}\left(1-\sqrt{\frac{1-\delta}{\mu}}\right)\right)\left\|\gamma f\left(x_{n}\right)-A q\right\|\left\|y_{n}-q\right\| \\
\leq & \alpha_{n}\left\|\gamma f\left(x_{n}\right)-A q\right\|^{2}+2 \alpha_{n}\left(1-\alpha_{n}\left(1-\sqrt{\frac{1-\delta}{\mu}}\right)\right)\left\|\gamma f\left(x_{n}\right)-A q\right\|\left\|y_{n}-q\right\| \\
& +\left(1-\alpha_{n}\left(1-\sqrt{\frac{1-\delta}{\mu}}\right)\left\{\left\|x_{n}-q\right\|^{2}+\lambda_{1}\left(\lambda_{1}-2 \beta_{1}\right)\left\|A_{1} u_{n}-A_{1} q\right\|^{2}\right\}\right. \\
& +\left\|x_{n}-q\right\|^{2}+\left(1-\alpha_{n}\left(1-\sqrt{\frac{1-\delta}{\mu}}\right)\right) \lambda_{1}\left(\lambda_{1}-2 \beta_{1}\right)\left\|A_{1} u_{n}-A_{1} q\right\|^{2} . \\
\leq & \alpha_{n}\left\|\gamma f\left(x_{n}\right)-A q\right\|^{2}+2 \alpha_{n}\left(1-\alpha_{n}\left(1-\sqrt{\frac{1-\delta}{\mu}}\right)\right)\left\|\gamma f\left(x_{n}\right)-A q\right\|\left\|y_{n}-q\right\| \\
& (1-1)
\end{aligned}
$$

By the convexity of the norm $\|\cdot\|$, we have

$$
\begin{aligned}
\left\|x_{n+1}-q\right\|^{2} & =\left\|\xi_{n} z_{n}+\left(1-\xi_{n}\right) P_{C} w_{n}-q\right\|^{2} \\
& =\left\|\xi_{n}\left(z_{n}-q\right)+\left(1-\xi_{n}\right)\left(P_{C} w_{n}-q\right)\right\|^{2} \\
& \leq \xi_{n}\left\|z_{n}-q\right\|^{2}+\left(1-\xi_{n}\right)\left\|w_{n}-q\right\|^{2} .
\end{aligned}
$$

Substituting (3.4), (3.7), (3.24) into (3.25), we obtain

$$
\begin{aligned}
\left\|x_{n+1}-q\right\|^{2} & \\
\leq & \xi_{n}\left\{\alpha_{n}\left\|\gamma f\left(x_{n}\right)-A q\right\|^{2}+2 \alpha_{n}\left(1-\alpha_{n}\left(1-\sqrt{\frac{1-\delta}{\mu}}\right)\right)\right. \\
& \times\left\|\gamma f\left(x_{n}\right)-A q\right\|\left\|y_{n}-q\right\|+\left\|x_{n}-q\right\|^{2}+\left(1-\alpha_{n}\left(1-\sqrt{\frac{1-\delta}{\mu}}\right)\right) \\
& \left.\times \lambda_{1}\left(\lambda_{1}-2 \beta_{1}\right)\left\|A_{1} u_{n}-A_{1} q\right\|^{2}\right\}+\left(1-\xi_{n}\right)\left\|x_{n}-q\right\|^{2} \\
= & \xi_{n} \alpha_{n}\left\|\gamma f\left(x_{n}\right)-A q\right\|^{2}+2 \xi_{n} \alpha_{n}\left(1-\alpha_{n}\left(1-\sqrt{\frac{1-\delta}{\mu}}\right)\right) \\
& \times\left\|\gamma f\left(x_{n}\right)-A q\right\|\left\|y_{n}-q\right\|+\xi_{n}\left\|x_{n}-q\right\|^{2}+\xi_{n}\left(1-\alpha_{n}\left(1-\sqrt{\frac{1-\delta}{\mu}}\right)\right) \\
& \times \lambda_{1}\left(\lambda_{1}-2 \beta_{1}\right)\left\|A_{1} u_{n}-A_{1} q\right\|^{2}+\left(1-\xi_{n}\right)\left\|x_{n}-q\right\|^{2} .
\end{aligned}
$$

So, we obtain

$$
\begin{aligned}
& \xi_{n}\left(1-\alpha_{n}\left(1-\frac{1-\delta}{\mu}\right)\right) \lambda_{1}\left(2 \beta_{1}-\lambda_{1}\right)\left\|A_{1} u_{n}-A_{1} q\right\|^{2} \\
& \quad \leq \xi_{n} \alpha_{n}\left\|\gamma f\left(x_{n}\right)-A q\right\|^{2}+\varepsilon_{n}+\left\|x_{n}-x_{n+1}\right\|\left(\left\|x_{n}-q\right\|+\left\|x_{n+1}-q\right\|\right),
\end{aligned}
$$


where $\varepsilon_{n}=2 \xi_{n} \alpha_{n}\left(1-\alpha_{n}\left(1-\sqrt{\frac{1-\delta}{\mu}}\right)\right)\left\|\gamma f\left(x_{n}\right)-A q\right\|\left\|y_{n}-q\right\|$. Since conditions (C1), (C2) and $\lim _{n \rightarrow \infty}|| x_{n+1}-x_{n} \|=0$, then we obtain that $\left\|A_{1} u_{n}-A_{1} q\right\| \rightarrow 0$ as $n \rightarrow$ $\infty$. For $q \in \Theta$ and $q=J_{M_{2}, \lambda_{2}}\left(q-\lambda_{2} A_{2} q\right)$, then we get

$$
\begin{aligned}
\left\|w_{n}-q\right\|^{2} & =\left\|J_{M_{2}, \lambda_{2}}\left(v_{n}-\lambda_{2} A_{2} v_{n}\right)-J_{M_{2}, \lambda_{2}}\left(q-\lambda_{2} A_{2} q\right)\right\|^{2} \\
& \leq\left\|\left(v_{n}-\lambda_{2} A_{2} v_{n}\right)-\left(q-\lambda_{2} A_{2} q\right)\right\|^{2} \\
& \leq\left\|v_{n}-q\right\|^{2}+\lambda_{2}\left(\lambda_{2}-2 \beta_{2}\right)\left\|A_{2} v_{n}-A_{2} q\right\|^{2} \\
& \leq\left\|x_{n}-q\right\|^{2}+\lambda_{2}\left(\lambda_{2}-2 \beta_{2}\right)\left\|A_{2} v_{n}-A_{2} q\right\|^{2}
\end{aligned}
$$

Substituting (3.24), (3.26) into (3.25), we obtain

$$
\begin{aligned}
\left\|x_{n+1}-q\right\|^{2} & \\
\leq & \xi_{n}\left\{\alpha_{n}\left\|\gamma f\left(x_{n}\right)-A q\right\|^{2}+2 \alpha_{n}\left(1-\alpha_{n}\left(1-\sqrt{\frac{1-\delta}{\mu}}\right)\right)\left\|\gamma f\left(x_{n}\right)-A q\right\|\left\|y_{n}-q\right\|\right. \\
& \left.+\left\|x_{n}-q\right\|^{2}+\left(1-\alpha_{n}\left(1-\sqrt{\frac{1-\delta}{\mu}}\right)\right) \lambda_{1}\left(\lambda_{1}-2 \beta_{1}\right)\left\|A_{1} u_{n}-A_{1} q\right\|^{2}\right\} \\
& +\left(1-\xi_{n}\right)\left\{\left\|x_{n}-q\right\|^{2}+\lambda_{2}\left(\lambda_{2}-2 \beta_{2}\right)\left\|A_{2} v_{n}-A_{2} q\right\|^{2}\right\} \\
= & \xi_{n} \alpha_{n}\left\|\gamma f\left(x_{n}\right)-A q\right\|^{2}+2 \xi_{n} \alpha_{n}\left(1-\alpha_{n}\left(1-\sqrt{\frac{1-\delta}{\mu}}\right)\right)\left\|\gamma f\left(x_{n}\right)-A q\right\|\left\|y_{n}-q\right\| \\
& +\xi_{n}\left\|x_{n}-q\right\|^{2}+\xi_{n}\left(1-\alpha_{n}\left(1-\sqrt{\frac{1-\delta}{\mu}}\right)\right) \lambda_{1}\left(\lambda_{1}-2 \beta_{1}\right)\left\|A_{1} u_{n}-A_{1} q\right\|^{2} \\
& +\left(1-\xi_{1}\right)\left\|x_{n}-q\right\|^{2}+\left(1-\xi_{n}\right) \lambda_{2}\left(\lambda_{2}-2 \beta_{2}\right)\left\|A_{2} v_{n}-A_{2} q\right\|^{2} .
\end{aligned}
$$

So, we obtain

$$
\begin{aligned}
& \left(1-\xi_{n}\right) \lambda_{2}\left(2 \beta_{2}-\lambda_{2}\right)\left\|A_{2} v_{n}-A_{2} q\right\|^{2} \\
& \leq \xi_{n} \alpha_{n}\left\|\gamma f\left(x_{n}\right)-A q\right\|^{2}+\varepsilon_{n}+\xi_{n}\left(1-\alpha_{n}\left(1-\sqrt{\frac{1-\delta}{\mu}}\right)\right) \lambda_{1}\left(\lambda_{1}-2 \beta_{1}\right) \\
& \quad \times\left\|A_{1} u_{n}-A_{1} q\right\|^{2}+\left\|x_{n}-x_{n+1}\right\|\left(\left\|x_{n}-q\right\|+\left\|x_{n+1}-q\right\|\right),
\end{aligned}
$$

where $\varepsilon_{n}=2 \xi_{n} \alpha_{n}\left(1-\alpha_{n}\left(1-\sqrt{\frac{1-\delta}{\mu}}\right)\right)\left\|\gamma f\left(x_{n}\right)-A q\right\|\left\|y_{n}-q\right\|$. Since conditions (C1), (C2), $\lim _{n \rightarrow \infty}|| x_{n+1}-x_{n}||=0$ and $\lim _{n \rightarrow \infty}|| A_{1} u_{n}-A_{1} q \| \rightarrow 0$ then we obtain that $\left\|A_{2} v_{n}-A_{2} q\right\| \rightarrow 0$ as $n \rightarrow \infty$. We consider this inequality in (3.24) that

$$
\begin{aligned}
\left\|z_{n}-q\right\|^{2} \leq & \alpha_{n}\left\|\gamma f\left(x_{n}\right)-A q\right\|^{2}+\left(1-\alpha_{n}\left(1-\sqrt{\frac{1-\delta}{\mu}}\right)\right)\left\|y_{n}-q\right\|^{2} \\
& +2 \alpha_{n}\left(1-\alpha_{n}\left(1-\sqrt{\frac{1-\delta}{\mu}}\right)\right)\left\|\gamma f\left(x_{n}\right)-A q\right\|\left\|y_{n}-q\right\| .
\end{aligned}
$$


Substituting (3.3) and (3.6) into (3.27), we have

$$
\begin{aligned}
\left\|z_{n}-q\right\|^{2} \leq & \alpha_{n}\left\|\gamma f\left(x_{n}\right)-A q\right\|^{2}+\left(1-\alpha_{n}\left(1-\sqrt{\frac{1-\delta}{\mu}}\right)\right) \\
& \times\left\{\left\|x_{n}-q\right\|^{2}+r_{n}\left(r_{n}-2 \eta\right)\left\|B_{1} x_{n}-B_{1} q\right\|^{2}\right\} \\
& +2 \alpha_{n}\left(1-\alpha_{n}\left(1-\sqrt{\frac{1-\delta}{\mu}}\right)\right)\left\|\gamma f\left(x_{n}\right)-A q\right\|\left\|y_{n}-q\right\| \\
= & \alpha_{n}\left\|\gamma f\left(x_{n}\right)-A q\right\|^{2}+\left(1-\alpha_{n}\left(1-\sqrt{\frac{1-\delta}{\mu}}\right)\right)\left\|x_{n}-q\right\|^{2} \\
& +\left(1-\alpha_{n}\left(1-\sqrt{\frac{1-\delta}{\mu}}\right)\right) r_{n}\left(r_{n}-2 \eta\right)\left\|B_{1} x_{n}-B_{1} q\right\|^{2} \\
& +2 \alpha_{n}\left(1+\alpha_{n}\left(1-\sqrt{\frac{1-\delta}{\mu}}\right)\right)\left\|\gamma f\left(x_{n}\right)-A q\right\|\left\|y_{n}-q\right\| \\
\leq & \alpha_{n}\left\|\gamma f\left(x_{n}\right)-A q\right\|^{2}+\left\|x_{n}-q\right\|^{2} \\
& +\left(1-\alpha_{n}\left(1-\sqrt{\frac{1-\delta}{\mu}}\right)\right) r_{n}\left(r_{n}-2 \eta\right)\left\|B_{1} x_{n}-B_{1} q\right\|^{2} \\
& +2 \alpha_{n}\left(1-\alpha_{n}\left(1-\sqrt{\frac{1-\delta}{\mu}}\right)\right)\left\|\gamma f\left(x_{n}\right)-A q\right\|\left\|y_{n}-q\right\| .
\end{aligned}
$$

Substituting (3.4), (3.7) and (3.28) into (3.25), we obtain

$$
\begin{aligned}
& \left\|x_{n+1}-q\right\|^{2} \\
& \leq \xi_{n}\left\{\alpha_{n}\left\|\gamma f\left(x_{n}\right)-A q\right\|^{2}+\left\|x_{n}-q\right\|^{2}+\left(1-\alpha_{n}\left(1-\sqrt{\frac{1-\delta}{\mu}}\right)\right)\right. \\
& \quad \times r_{n}\left(r_{n}-2 \eta\right)\left\|B_{1} x_{n}-B_{1} q\right\|^{2}+2 \alpha_{n}\left(1-\alpha_{n}\left(1-\sqrt{\frac{1-\delta}{\mu}}\right)\right) \\
& \left.\quad \times\left\|\gamma f\left(x_{n}\right)-A q\right\|\left\|y_{n}-q\right\|\right\}+\left(1-\xi_{n}\right)\left\|x_{n}-q\right\|^{2} \\
& =\xi_{n} \alpha_{n}\left\|\gamma f\left(x_{n}\right)-A q\right\|^{2}+\xi_{n}\left\|x_{n}-q\right\|^{2}+\xi_{n}\left(1-\alpha_{n}\left(1-\sqrt{\frac{1-\delta}{\mu}}\right)\right) \\
& \quad \times r_{n}\left(r_{n}-2 \eta\right)\left\|B_{1} x_{n}-B_{1} q\right\|^{2}+2 \xi_{n} \alpha_{n}\left(1-\alpha_{n}\left(1-\sqrt{\frac{1-\delta}{\mu}}\right)\right) \\
& \quad \times\left\|\gamma f\left(x_{n}\right)-A q\right\|\left\|y_{n}-q\right\|+\left(1-\xi_{n}\right)\left\|x_{n}-q\right\|^{2} .
\end{aligned}
$$

So, we also have

$$
\begin{aligned}
& \xi_{n}\left(1-\alpha_{n}\left(1-\sqrt{\frac{1-\delta}{\mu}}\right)\right) r_{n}\left(2 \eta-r_{n}\right)\left\|B_{1} x_{n}-B_{1} q\right\|^{2} \\
& \quad \leq \xi_{n} \alpha_{n}\left\|\gamma f\left(x_{n}\right)-A q\right\|^{2}+\varepsilon_{n}+\left\|x_{n}-x_{n+1}\right\|\left(\left\|x_{n}-q\right\|+\left\|x_{n+1}-q\right\|\right),
\end{aligned}
$$


where $\varepsilon_{n}=2 \xi_{n} \alpha_{n}\left(1-\alpha_{n}\left(1-\sqrt{\frac{1-\delta}{\mu}}\right)\right)\left\|\gamma f\left(x_{n}\right)-A q\right\|\left\|y_{n}-q\right\|$. Since conditions (C1), (C2), (C3), $\lim _{n \rightarrow \infty}\left\|x_{n+1}-x_{n}\right\|=0$, then we obtain that $\left\|B_{1} x_{n}-B_{1} q\right\| \rightarrow 0$ as $n$ $\rightarrow \infty$. Substituting (3.4), (3.7) and (3.28) into (3.25), we obtain

$$
\begin{aligned}
&\left\|x_{n+1}-q\right\|^{2} \\
& \leq \xi_{n}\left\{\alpha_{n}\left\|\gamma f\left(x_{n}\right)-A q\right\|^{2}+\left\|x_{n}-q\right\|^{2}+\left(1-\alpha_{n}\left(1-\sqrt{\frac{1-\delta}{\mu}}\right)\right)\right. \\
& \times r_{n}\left(r_{n}-2 \eta\right)\left\|B_{1} x_{n}-B_{1} q\right\|^{2}+2 \alpha_{n}\left(1-\alpha_{n}\left(1-\sqrt{\frac{1-\delta}{\mu}}\right)\right) \\
&\left.\times\left\|\gamma f\left(x_{n}\right)-A q\right\|\left\|y_{n}-q\right\|\right\} \\
&+\left(1-\xi_{n}\right)\left\{\left\|x_{n}-q\right\|^{2}+s_{n}\left(s_{n}-2 \rho\right)\left\|B_{2} x_{n}-B_{2} q\right\|^{2}\right\} \\
&= \xi_{n} \alpha_{n}\left\|\gamma f\left(x_{n}\right)-A q\right\|^{2}+\xi_{n}\left\|x_{n}-q\right\|^{2}+\xi_{n}\left(1-\alpha_{n}\left(1-\sqrt{\frac{1-\delta}{\mu}}\right)\right) \\
& \times r_{n}\left(r_{n}-2 \eta\right)\left\|B_{1} x_{n}-B_{1} q\right\|^{2}+2 \xi_{n} \alpha_{n}\left(1-\alpha_{n}\left(1-\sqrt{\frac{1-\delta}{\mu}}\right)\right) \\
& \times\left\|\gamma f\left(x_{n}\right)-A q\right\|\left\|y_{n}-q\right\|+\left(1-\xi_{n}\right)\left\|x_{n}-q\right\|^{2} \\
&+\left(1-\xi_{n}\right) s_{n}\left(s_{n}-2 \rho\right)\left\|B_{2} x_{n}-B_{2} q\right\|^{2} \\
&= \xi_{n} \alpha_{n}\left\|\gamma f\left(x_{n}\right)-A q\right\|^{2}+\left\|x_{n}-q\right\|^{2}+\xi_{n}\left(1-\alpha_{n}\left(1-\sqrt{\frac{1-\delta}{\mu}}\right)\right) \\
& \times r_{n}\left(r_{n}-2 \eta\right)\left\|B_{1} x_{n}-B_{1} q\right\|^{2}+2 \xi_{n} \alpha_{n}\left(1-\alpha_{n}\left(1-\sqrt{\frac{1-\delta}{\mu}}\right)\right) \\
& \times\left\|\gamma f\left(x_{n}\right)-A q\right\|\left\|y_{n}-q\right\| \\
&+\left(1-\xi_{n}\right) s_{n}\left(s_{n}-2 \rho\right)\left\|B_{2} x_{n}-B_{2} q\right\|^{2} .
\end{aligned}
$$

So, we also have

$$
\begin{aligned}
& \left(1-\xi_{n}\right) s_{n}\left(2 \rho-s_{n}\right)\left\|B_{2} x_{n}-B_{2} q\right\|^{2} \\
& \leq \xi_{n} \alpha_{n}\left\|\gamma f\left(x_{n}\right)-A q\right\|^{2}+\varepsilon_{n}+\left\|x_{n}-x_{n+1}\right\|\left(\left\|x_{n}-q\right\|+\left\|x_{n+1}-q\right\|\right) \\
& \quad+\xi_{n}\left(1-\alpha_{n}\left(1-\sqrt{\frac{1-\delta}{\mu}}\right)\right) r_{n}\left(r_{n}-2 \eta\right)\left\|B_{1} x_{n}-B_{1} q\right\|^{2}
\end{aligned}
$$

where $\varepsilon_{n}=2 \xi_{n} \alpha_{n}\left(1-\alpha_{n}\left(1-\sqrt{\frac{1-\delta}{\mu}}\right)\right)\left\|\gamma f\left(x_{n}\right)-A q\right\|\left\|\gamma_{n}-q\right\|$. Since conditions (C1), (C2), (C4), $\lim _{n \rightarrow \infty}|| x_{n+1}-x_{n}||=0$ and $\lim _{n \rightarrow \infty}|| B_{1} x_{n}-B_{1} q \|=0$, then we obtain that $\left\|B_{2} x_{n}-B_{2} q\right\| \rightarrow 0$ as $n \rightarrow \infty$.

Step 4. We show the followings:
(i) $\lim _{n \rightarrow \infty}|| x_{n}-u_{n} \|=0$;
(ii) $\lim _{n \rightarrow \infty}\left\|u_{n}-y_{n}\right\|=0$;
(iii) $\lim _{n \rightarrow \infty}|| x_{n}-v_{n} \|=0$;
(iv) $\lim _{n \rightarrow \infty}|| v_{n}-w_{n} \|=0$; 
(v) $\lim _{n \rightarrow \infty}\left\|u_{n}-y_{n}^{\prime}\right\|=0$;

(vi) $\lim _{n \rightarrow \infty}\left\|y_{n}^{\prime}-S y_{n}^{\prime}\right\|=0$.

Since $T_{r_{n}}^{\left(F_{1}, \varphi_{1}\right)}$ is firmly nonexpansive, we observe that

$$
\begin{aligned}
&\left\|u_{n}-q\right\|^{2} \\
&=\left\|T_{r_{n}}^{\left(F_{1}, \varphi_{1}\right)}\left(x_{n}-r_{n} B_{1} x_{n}\right)-T_{r_{n}}^{\left(F_{1}, \varphi_{1}\right)}\left(q-r_{n} B_{1} q\right)\right\|^{2} \\
& \leq\left\langle\left(x_{n}-r_{n} B_{1} x_{n}\right)-\left(q-r_{n} B_{1} q\right), u_{n}-q\right) \\
&= \frac{1}{2}\left(\left\|\left(x_{n}-r_{n} B_{1} x_{n}\right)-\left(q-r_{n} B_{1} q\right)\right\|^{2}+\left\|u_{n}-q\right\|^{2}-\|\left(x_{n}-r_{n} B_{1} x_{n}\right)\right. \\
&\left.-\left(q-r_{n} B_{1} q\right)-\left(u_{n}-q\right) \|^{2}\right) \\
& \leq \frac{1}{2}\left(\left\|x_{n}-q\right\|^{2}+\left\|u_{n}-q\right\|^{2}-\left\|\left(x_{n}-u_{n}\right)-r_{n}\left(B_{1} x_{n}-B_{1} q\right)\right\|^{2}\right) \\
&= \frac{1}{2}\left(\left\|x_{n}-q\right\|^{2}+\left\|u_{n}-q\right\|^{2}-\left\|x_{n}-u_{n}\right\|^{2}\right. \\
&\left.+2 r_{n}\left\langle B_{1} x_{n}-B_{1} q, x_{n}-u_{n}\right\rangle-r_{n}^{2}\left\|B_{1} x_{n}-B_{1} q\right\|^{2}\right) .
\end{aligned}
$$

Hence, we have

$$
\left\|u_{n}-q\right\|^{2} \leq\left\|x_{n}-q\right\|^{2}-\left\|x_{n}-u_{n}\right\|^{2}+2 r_{n}\left\|B_{1} x_{n}-B_{1} q\right\|\left\|x_{n}-u_{n}\right\| .
$$

Since $J_{M_{1}, \lambda_{1}}$ is 1-inverse-strongly monotone, we compute

$$
\begin{aligned}
\left\|y_{n}-q\right\|^{2}= & \left\|J_{M_{1}, \lambda_{1}}\left(u_{n}-\lambda_{1} A_{1} u_{n}\right)-J_{M_{1}, \lambda_{1}}\left(q-\lambda_{1} A_{1} q\right)\right\|^{2} \\
\leq & \left\langle\left(u_{n}-\lambda_{1} A_{1} u_{n}\right)-\left(q-\lambda_{1} A_{1} q\right), y_{n}-q\right\rangle \\
= & \frac{1}{2}\left(\left\|\left(u_{n}-\lambda_{1} A_{1} u_{n}\right)-\left(q-\lambda_{1} A_{1} q\right)\right\|^{2}+\left\|y_{n}-q\right\|^{2}\right. \\
& -\left\|\left(u_{n}-\lambda_{1} A_{1} u_{n}\right)-\left(q-\lambda_{1} A_{1} q\right)-\left(y_{n}-q\right)\right\|^{2} \\
= & \frac{1}{2}\left(\left\|u_{n}-q\right\|^{2}+\left\|y_{n}-q\right\|^{2}-\left\|\left(u_{n}-y_{n}\right)-\lambda_{1}\left(A_{1} u_{n}-A_{1} q\right)\right\|^{2}\right) \\
\leq & \frac{1}{2}\left(\left\|u_{n}-q\right\|^{2}+\left\|y_{n}-q\right\|^{2}-\left\|u_{n}-y_{n}\right\|^{2}\right. \\
& \left.+2 \lambda_{1}\left\langle u_{n}-y_{n}, A_{1} u_{n}-A_{1} q\right\rangle-\lambda_{1}^{2}\left\|A_{1} u_{n}-A_{1} q\right\|^{2}\right),
\end{aligned}
$$

which implies that

$$
\left\|y_{n}-q\right\|^{2} \leq\left\|u_{n}-q\right\|^{2}-\left\|u_{n}-y_{n}\right\|^{2}+2 \lambda_{1}\left\|u_{n}-y_{n}\right\|\left\|A_{1} u_{n}-A_{1} q\right\| .
$$

Substitute (3.30) into (3.31), we have

$$
\begin{aligned}
\left\|y_{n}-q\right\|^{2} \leq & \left\{\left\|x_{n}-q\right\|^{2}-\left\|x_{n}-u_{n}\right\|^{2}+2 r_{n}\left\|B_{1} x_{n}-B_{1} q\right\|\left\|x_{n}-u_{n}\right\|\right\} \\
& -\left\|u_{n}-y_{n}\right\|^{2}+2 \lambda_{1}\left\|u_{n}-y_{n}\right\|\left\|A_{1} u_{n}-A_{1} q\right\| .
\end{aligned}
$$


Substitute (3.32) into (3.27), we have

$$
\begin{aligned}
\left\|z_{n}-q\right\|^{2} \leq & \alpha_{n}\left\|\gamma f\left(x_{n}\right)-A q\right\|^{2}+\left(1-\alpha_{n}\left(1-\sqrt{\frac{1-\delta}{\mu}}\right)\right)\left\{\left\|x_{n}-q\right\|^{2}-\left\|x_{n}-u_{n}\right\|^{2}\right. \\
& \left.+2 r_{n}\left\|B_{1} x_{n}-B_{1} q\right\|\left\|x_{n}-u_{n}\right\|-\left\|u_{n}-\gamma_{n}\right\|^{2}+2 \lambda_{1}\left\|u_{n}-y_{n}\right\|\left\|A_{1} u_{n}-A_{1} q\right\|\right\} \\
& +2 \alpha_{n}\left(1-\alpha_{n}\left(1-\sqrt{\frac{1-\delta}{\mu}}\right)\right)\left\|\gamma f\left(x_{n}\right)-A q\right\|\left\|y_{n}-q\right\| \\
\leq & \alpha_{n}\left\|\gamma f\left(x_{n}\right)-A q\right\|^{2}+\left\|x_{n}-q\right\|^{2}-\left\|x_{n}-u_{n}\right\|^{2} \\
& +2\left(1-\alpha_{n}\left(1-\sqrt{\frac{1-\delta}{\mu}}\right)\right) r_{n}\left\|B_{1} x_{n}-B_{1} q\right\|\left\|x_{n}-u_{n}\right\|-\left\|u_{n}-y_{n}\right\|^{2} \\
& +2\left(1-\alpha_{n}\left(1-\sqrt{\frac{1-\delta}{\mu}}\right)\right) \lambda_{1}\left\|u_{n}-y_{n}\right\|\left\|A_{1} u_{n}-A_{1} q\right\| \\
& +2 \alpha_{n}\left(1-\alpha_{n}\left(1-\sqrt{\frac{1-\delta}{\mu}}\right)\right)\left\|\gamma f\left(x_{n}\right)-A q\right\|\left\|y_{n}-q\right\| .
\end{aligned}
$$

Since $T_{s_{n}}^{\left(F_{2}, \varphi_{2}\right)}$ is firmly nonexpansive, we observe that

$$
\begin{aligned}
&\left\|v_{n}-q\right\|^{2} \\
&=\left\|T_{s_{n}}^{\left(F_{2}, \varphi_{2}\right)}\left(x_{n}-s_{n} B_{2} x_{n}\right)-T_{s_{n}}^{\left(F_{2}, \varphi_{2}\right)}\left(q-s_{n} B_{2} q\right)\right\|^{2} \\
& \leq\left\langle\left(x_{n}-s_{n} B_{2} x_{n}\right)-\left(q-s_{n} B_{2} q\right), v_{n}-q\right) \\
&= \frac{1}{2}\left(\left\|\left(x_{n}-s_{n} B_{2} x_{n}\right)-\left(q-s_{n} B_{2} q\right)\right\|^{2}+\left\|v_{n}-q\right\|^{2}\right. \\
&\left.-\left\|\left(x_{n}-s_{n} B_{2} x_{n}\right)-\left(q-s_{n} B_{2} q\right)-\left(v_{n}-q\right)\right\|^{2}\right) \\
& \leq \frac{1}{2}\left(\left\|x_{n}-q\right\|^{2}+\left\|v_{n}-q\right\|^{2}-\left\|\left(x_{n}-v_{n}\right)-s_{n}\left(B_{2} x_{n}-B_{2} q\right)\right\|^{2}\right) \\
&= \frac{1}{2}\left(\left\|x_{n}-q\right\|^{2}+\left\|v_{n}-q\right\|^{2}-\left\|x_{n}-v_{n}\right\|^{2}\right. \\
&\left.+2 s_{n}\left\langle B_{2} x_{n}-B_{2} q, x_{n}-v_{n}\right\rangle-s_{n}^{2}\left\|B_{2} x_{n}-B_{2} q\right\|^{2}\right) .
\end{aligned}
$$

Hence, we have

$$
\left\|v_{n}-q\right\|^{2} \leq\left\|x_{n}-q\right\|^{2}-\left\|x_{n}-v_{n}\right\|^{2}+2 s_{n}\left\|B_{2} x_{n}-B_{2} q\right\|\left\|x_{n}-v_{n}\right\| .
$$

Since $J_{M_{2}, \lambda_{2}}$ is 1-inverse-strongly monotone, we compute

$$
\begin{aligned}
\left\|w_{n}-q\right\|^{2}= & \left\|J_{M_{2}, \lambda_{2}}\left(v_{n}-\lambda_{2} A_{2} v_{n}\right)-J_{M_{2}, \lambda_{2}}\left(q-\lambda_{2} A_{2} q\right)\right\|^{2} \\
\leq & \left\langle\left(v_{n}-\lambda_{2} A_{2} v_{n}\right)-\left(q-\lambda_{2} A_{2} q\right), w_{n}-q\right) \\
= & \frac{1}{2}\left(\left\|\left(v_{n}-\lambda_{2} A_{2} v_{n}\right)-\left(q-\lambda_{2} A_{2} q\right)\right\|^{2}+\left\|w_{n}-q\right\|^{2}\right. \\
& \left.-\left\|\left(v_{n}-\lambda_{2} A_{2} v_{n}\right)-\left(q-\lambda_{2} A_{2} q\right)-\left(w_{n}-q\right)\right\|^{2}\right) \\
= & \frac{1}{2}\left(\left\|v_{n}-q\right\|^{2}+\left\|w_{n}-q\right\|^{2}-\left\|\left(v_{n}-w_{n}\right)-\lambda_{2}\left(A_{2} v_{n}-A_{2} q\right)\right\|^{2}\right) \\
\leq & \frac{1}{2}\left(\left\|v_{n}-q\right\|^{2}+\left\|w_{n}-q\right\|^{2}-\left\|v_{n}-w_{n}\right\|^{2}\right. \\
& \left.+2 \lambda_{2}\left\langle v_{n}-w_{n}, A_{2} v_{n}-A_{2} q\right\rangle-\lambda_{2}^{2}\left\|A_{2} v_{n}-A_{2} q\right\|^{2}\right),
\end{aligned}
$$

which implies that

$$
\left\|w_{n}-q\right\|^{2} \leq\left\|v_{n}-q\right\|^{2}-\left\|v_{n}-w_{n}\right\|^{2}+2 \lambda_{2}\left\|v_{n}-w_{n}\right\|\left\|A_{2} v_{n}-A_{2} q\right\| .
$$


Substitute (3.34) into (3.35), we have

$$
\begin{aligned}
\left\|w_{n}-q\right\|^{2} \leq & \left\{\left\|x_{n}-q\right\|^{2}-\left\|x_{n}-v_{n}\right\|^{2}+2 s_{n}\left\|B_{2} x_{n}-B_{2} q\right\|\left\|x_{n}-v_{n}\right\|\right\} \\
& -\left\|v_{n}-w_{n}\right\|^{2}+2 \lambda_{2}\left\|v_{n}-w_{n}\right\|\left\|A_{2} v_{n}-A_{2} q\right\| .
\end{aligned}
$$

Substitute (3.33) and (3.36) into (3.25), we obtain

$$
\begin{aligned}
\left\|x_{n+1}-q\right\|^{2} \leq & \xi_{n}\left\|z_{n}-q\right\|^{2}+\left(1-\xi_{n}\right)\left\|w_{n}-q\right\|^{2} \\
\leq & \xi_{n}\left\{\alpha_{n}\left\|\gamma f\left(x_{n}\right)-A q\right\|^{2}+\left\|x_{n}-q\right\|^{2}-\left\|x_{n}-u_{n}\right\|^{2}-\left\|u_{n}-y_{n}\right\|^{2}\right. \\
& +2\left(1-\alpha_{n}\left(1-\sqrt{\frac{1-\delta}{\mu}}\right)\right) r_{n}\left\|B_{1} x_{n}-B_{1} q\right\|\left\|x_{n}-u_{n}\right\| \\
& +2\left(1-\alpha_{n}\left(1-\sqrt{\frac{1-\delta}{\mu}}\right)\right) \lambda_{1}\left\|u_{n}-\gamma_{n}\right\|\left\|A_{1} u_{n}-A_{1} q\right\| \\
& \left.+2 \alpha_{n}\left(1-\alpha_{n}\left(1-\sqrt{\frac{1-\delta}{\mu}}\right)\right)\left\|\gamma f\left(x_{n}\right)-A q\right\|\left\|y_{n}-q\right\|\right\} \\
& +\left(1-\xi_{n}\right)\left\{\left\|x_{n}-q\right\|^{2}-\left\|x_{n}-v_{n}\right\|^{2}\right. \\
& +2 s_{n}\left\|B_{2} x_{n}-B_{2} q\right\|\left\|x_{n}-v_{n}\right\|-\left\|v_{n}-w_{n}\right\|^{2} \\
& \left.+2 \lambda_{2}\left\|v_{n}-w_{n}\right\|\left\|A_{2} v_{n}-A_{2} q\right\|\right\} \\
\leq & \xi_{n} \alpha_{n}\left\|\gamma f\left(x_{n}\right)-A q\right\|^{2}+\xi_{n}\left\|x_{n}-q\right\|^{2}-\left\|x_{n}-u_{n}\right\|^{2}-\left\|u_{n}-y_{n}\right\|^{2} \\
& +2 \xi_{n}\left(1-\alpha_{n}\left(1-\sqrt{\frac{1-\delta}{\mu}}\right)\right) r_{n}\left\|B_{1} x_{n}-B_{1} q\right\|\left\|x_{n}-u_{n}\right\| \\
& +2 \xi_{n}\left(1-\alpha_{n}\left(1-\sqrt{\frac{1-\delta}{\mu}}\right)\right) \lambda_{1}\left\|u_{n}-y_{n}\right\|\left\|A_{1} u_{n}-A_{1} q\right\| \\
& +2 \xi_{n} \alpha_{n}\left(1-\alpha_{n}\left(1-\sqrt{\frac{1-\delta}{\mu}}\right)\right)\left\|\gamma f\left(x_{n}\right)-A q\right\|\left\|y_{n}-q\right\| \\
& +\left(1-\xi_{n}\right)\left\|x_{n}-q\right\|^{2}-\left\|x_{n}-v_{n}\right\|^{2}+2\left(1-\xi_{n}\right) s_{n}\left\|B_{2} x_{n}-B_{2} q\right\|\left\|x_{n}-v_{n}\right\| \\
& -\left\|v_{n}-w_{n}\right\|^{2}+2\left(1-\xi_{n}\right) \lambda_{2}\left\|v_{n}-w_{n}\right\|\left\|A_{2} v_{n}-A_{2} q\right\| . \\
& \\
&
\end{aligned}
$$

Then, we derive

$$
\begin{aligned}
\| x_{n}- & u_{n}\left\|^{2}+\right\| u_{n}-y_{n}\left\|^{2}+\right\| x_{n}-v_{n}\left\|^{2}+\right\| v_{n}-w_{n} \|^{2} \\
\leq & \xi_{n} \alpha_{n}\left\|\gamma f\left(x_{n}\right)-A q\right\|^{2}+\left\|x_{n}-q\right\|^{2}-\left\|x_{n+1}-q\right\|^{2} \\
& +2 \xi_{n}\left(1-\alpha_{n}\left(1-\sqrt{\frac{1-\delta}{\mu}}\right)\right) r_{n}\left\|B_{1} x_{n}-B_{1} q\right\|\left\|x_{n}-u_{n}\right\| \\
& +2 \xi_{n}\left(1-\alpha_{n}\left(1-\sqrt{\frac{1-\delta}{\mu}}\right)\right) \lambda_{1}\left\|u_{n}-y_{n}\right\|\left\|A_{1} u_{n}-A_{1} q\right\| \\
& +2 \xi_{n} \alpha_{n}\left(1-\alpha_{n}\left(1-\sqrt{\frac{1-\delta}{\mu}}\right)\right)\left\|\gamma f\left(x_{n}\right)-A q\right\|\left\|y_{n}-q\right\| \\
& +2\left(1-\xi_{n}\right) s_{n}\left\|B_{2} x_{n}-B_{2} q\right\|\left\|x_{n}-v_{n}\right\|+2\left(1-\xi_{n}\right) \lambda_{2}\left\|v_{n}-w_{n}\right\|\left\|A_{2} v_{n}-A_{2} q\right\| \\
= & \xi_{n} \alpha_{n}\left\|\gamma f\left(x_{n}\right)-A q\right\|^{2}+\left\|x_{n+1}-x_{n}\right\|\left(\left\|x_{n}-q\right\|+\left\|x_{n+1}-q\right\|\right) \\
& +2 \xi_{n}\left(1-\alpha_{n}\left(1-\sqrt{\frac{1-\delta}{\mu}}\right)\right) r_{n}\left\|B_{1} x_{n}-B_{1} q\right\|\left\|x_{n}-u_{n}\right\| \\
& +2 \xi_{n}\left(1-\alpha_{n}\left(1-\sqrt{\frac{1-\delta}{\mu}}\right)\right) \lambda_{1}\left\|u_{n}-y_{n}\right\|\left\|A_{1} u_{n}-A_{1} q\right\| \\
& +2 \xi_{n} \alpha_{n}\left(1-\alpha_{n}\left(1-\sqrt{\frac{1-\delta}{\mu}}\right)\right)\left\|\gamma f\left(x_{n}\right)-A q\right\|\left\|y_{n}-q\right\| \\
& +2\left(1-\xi_{n}\right) s_{n}\left\|B_{2} x_{n}-B_{2} q\right\|\left\|x_{n}-v_{n}\right\|+2\left(1-\xi_{n}\right) \lambda_{2}\left\|v_{n}-w_{n}\right\|\left\|A_{2} v_{n}-A_{2} q\right\| .
\end{aligned}
$$


By conditions (C1)-( C4), $\lim _{n \rightarrow \infty}\left\|x_{n}-x_{n+1}\right\|=0, \lim _{n \rightarrow \infty}\left\|B_{1} x_{n}-B_{1} q\right\|=0, \lim _{n \rightarrow \infty} \|$ $B_{2} x_{n}-B_{2} q\left\|=0, \lim _{n \rightarrow \infty}\right\| A_{1} u_{n}-A_{1} q \|=0$ and $\lim _{n \rightarrow \infty}\left\|A_{2} v_{n}-A_{2} q\right\|=0$. So, we have $\left\|x_{n}-u_{n}\right\| \rightarrow 0,\left\|u_{n}-y_{n}\right\| \rightarrow 0,\left\|x_{n}-v_{n}\right\| \rightarrow 0,\left\|v_{n}-w_{n}\right\| \rightarrow 0$ as $n \rightarrow \infty$.

From (2.1), we have

$$
\begin{aligned}
\left\|y_{n}^{\prime}-q\right\|^{2}= & \left\|P_{C} J_{M_{1} \lambda_{1}}\left(u_{n}-\lambda_{1} A_{1} u_{n}\right)-P_{C} J_{M_{1} \lambda_{1}}\left(q-\lambda_{1} A_{1} q\right)\right\|^{2} \\
\leq & \left\|J_{M_{1}, \lambda_{1}}\left(u_{n}-\lambda_{1} A_{1} u_{n}\right)-J_{M_{1}, \lambda_{1}}\left(q-\lambda_{1} A_{1} q\right)\right\|^{2} \\
\leq & \left\langle\left(u_{n}-\lambda_{1} A_{1} u_{n}\right)-\left(q-\lambda_{1} A_{1} q\right), \gamma_{n}^{\prime}-q\right\rangle \\
= & \frac{1}{2}\left(\left\|\left(u_{n}-\lambda_{1} A_{1} u_{n}\right)-\left(q-\lambda_{1} A_{1} q\right)\right\|^{2}+\left\|y_{n}^{\prime}-q\right\|^{2}\right. \\
& \left.-\left\|\left(u_{n}-\lambda_{1} A_{1} u_{n}\right)-\left(q-\lambda_{1} A_{1} q\right)-\left(\gamma_{n}^{\prime}-q\right)\right\|^{2}\right) \\
= & \frac{1}{2}\left(\left\|u_{n}-q\right\|^{2}+\left\|y_{n}^{\prime}-q\right\|^{2}-\left\|\left(u_{n}-\gamma_{n}^{\prime}\right)-\lambda_{1}\left(A_{1} u_{n}-A_{1} q\right)\right\|^{2}\right) \\
\leq & \frac{1}{2}\left(\left\|u_{n}-q\right\|^{2}+\left\|y_{n}^{\prime}-q\right\|^{2}-\left\|u_{n}-y_{n}^{\prime}\right\|^{2}\right. \\
& \left.+2 \lambda_{1}\left\langle u_{n}-\gamma_{n^{\prime}}{ }^{\prime} A_{1} u_{n}-A_{1} q\right\rangle-\lambda_{1}^{2}\left\|A_{1} u_{n}-A_{1} q\right\|^{2}\right),
\end{aligned}
$$

which implies that

$$
\left\|y_{n}^{\prime}-q\right\|^{2} \leq\left\|u_{n}-q\right\|^{2}-\left\|u_{n}-y_{n}^{\prime}\right\|^{2}+2 \lambda_{1}\left\|u_{n}-y_{n}^{\prime}\right\|\left\|A_{1} u_{n}-A_{1} q\right\| .
$$

Substitute (3.30) into (3.37), we have

$$
\begin{aligned}
\left\|y_{n}^{\prime}-q\right\|^{2} \leq & \left\{\left\|x_{n}-q\right\|^{2}-\left\|x_{n}-u_{n}\right\|^{2}+2 r_{n}\left\|B_{1} x_{n}-B_{1} q\right\|\left\|x_{n}-u_{n}\right\|\right\} \\
& -\left\|u_{n}-y^{\prime}{ }_{n}\right\|^{2}+2 \lambda_{1}\left\|u_{n}-\gamma_{n}^{\prime}\right\|\left\|A_{1} u_{n}-A_{1} q\right\| .
\end{aligned}
$$

We consider this inequality in (3.24) that

$$
\begin{aligned}
\left\|z_{n}-q\right\|^{2} \leq & \alpha_{n}\left\|\gamma f\left(x_{n}\right)-A q\right\|^{2}+\left(1-\alpha_{n}\left(1-\sqrt{\frac{1-\delta}{\mu}}\right)\right)\left\|\gamma_{n}^{\prime}-q\right\|^{2} \\
& +2 \alpha_{n}\left(1-\alpha_{n}\left(1-\sqrt{\frac{1-\delta}{\mu}}\right)\right)\left\|\gamma f\left(x_{n}\right)-A q\right\|\left\|y^{\prime}{ }_{n}-q\right\| .
\end{aligned}
$$

Substitute (3.38) into (3.39), we have

$$
\begin{aligned}
\left\|z_{n}-q\right\|^{2} \leq & \alpha_{n}\left\|\gamma f\left(x_{n}\right)-A q\right\|^{2}+\left(1-\alpha_{n}\left(1-\sqrt{\frac{1-\delta}{\mu}}\right)\right)\left\{\left\|x_{n}-q\right\|^{2}-\left\|x_{n}-u_{n}\right\|^{2}\right. \\
& \left.+2 r_{n}\left\|B_{1} x_{n}-B_{1} q\right\|\left\|x_{n}-u_{n}\right\|-\left\|u_{n}-\gamma_{n}^{\prime}\right\|^{2}+2 \lambda_{1}\left\|u_{n}-\gamma_{n}^{\prime}\right\|\left\|A_{1} u_{n}-A_{1} q\right\|\right\} \\
& +2 \alpha_{n}\left(1-\alpha_{n}\left(1-\sqrt{\frac{1-\delta}{\mu}}\right)\right)\left\|\gamma f\left(x_{n}\right)-A q\right\|\left\|\gamma^{\prime}{ }_{n}-q\right\| \\
\leq & \alpha_{n}\left\|\gamma f\left(x_{n}\right)-A q\right\|^{2}+\left\|x_{n}-q\right\|^{2}-\left\|x_{n}-u_{n}\right\|^{2} \\
& +2\left(1-\alpha_{n}\left(1-\sqrt{\frac{1-\delta}{\mu}}\right)\right) r_{n}\left\|B_{1} x_{n}-B_{1} q\right\|\left\|x_{n}-u_{n}\right\|-\left\|u_{n}-\gamma_{n}^{\prime}\right\|^{2} \\
& +2\left(1-\alpha_{n}\left(1-\sqrt{\frac{1-\delta}{\mu}}\right)\right) \lambda_{1}\left\|u_{n}-\gamma_{n}^{\prime}\right\|\left\|A_{1} u_{n}-A_{1} q\right\| \\
& +2 \alpha_{n}\left(1-\alpha_{n}\left(1-\sqrt{\frac{1-\delta}{\mu}}\right)\right)\left\|\gamma f\left(x_{n}\right)-A q\right\|\left\|y_{n}^{\prime}-q\right\| .
\end{aligned}
$$


Substitute (3.36) and (3.40) into (3.25), we obtain

$$
\begin{aligned}
\left\|x_{n+1}-q\right\|^{2} \leq & \xi_{n}\left\|z_{n}-q\right\|^{2}+\left(1-\xi_{n}\right)\left\|w_{n}-q\right\|^{2} \\
\leq & \xi_{n}\left\{\alpha_{n}\left\|\gamma f\left(x_{n}\right)-A q\right\|^{2}+\left\|x_{n}-q\right\|^{2}-\left\|x_{n}-u_{n}\right\|^{2}-\left\|u_{n}-\gamma_{n}^{\prime}\right\|^{2}\right. \\
& +2\left(1-\alpha_{n}\left(1-\sqrt{\frac{1-\delta}{\mu}}\right)\right) r_{n}\left\|B_{1} x_{n}-B_{1} q\right\|\left\|x_{n}-u_{n}\right\| \\
& +2\left(1-\alpha_{n}\left(1-\sqrt{\frac{1-\delta}{\mu}}\right)\right) \lambda_{1}\left\|u_{n}-\gamma_{n}^{\prime}\right\|\left\|A_{1} u_{n}-A_{1} q\right\| \\
& \left.+2 \alpha_{n}\left(1-\alpha_{n}\left(1-\sqrt{\frac{1-\delta}{\mu}}\right)\right)\left\|\gamma f\left(x_{n}\right)-A q\right\|\left\|\gamma_{n}^{\prime}-q\right\|\right\} \\
& +\left(1-\xi_{n}\right)\left\{\left\|x_{n}-q\right\|^{2}-\left\|x_{n}-v_{n}\right\|^{2}+2 s_{n}\left\|B_{2} x_{n}-B_{2} q\right\|\left\|x_{n}-v_{n}\right\|-\left\|v_{n}-w_{n}\right\|^{2}\right. \\
& \left.+2 \lambda_{2}\left\|v_{n}-w_{n}\right\|\left\|A_{2} v_{n}-A_{2} q\right\|\right\} \\
\leq & \xi_{n} \alpha_{n}\left\|\gamma f\left(x_{n}\right)-A q\right\|^{2}+\xi_{n}\left\|x_{n}-q\right\|^{2}-\left\|x_{n}-u_{n}\right\|^{2}-\left\|u_{n}-\gamma_{n}^{\prime}\right\|^{2} \\
& +2 \xi_{n}\left(1-\alpha_{n}\left(1-\sqrt{\frac{1-\delta}{\mu}}\right)\right) r_{n}\left\|B_{1} x_{n}-B_{1} q\right\|\left\|x_{n}-u_{n}\right\| \\
& +2 \xi_{n}\left(1-\alpha_{n}\left(1-\sqrt{\frac{1-\delta}{\mu}}\right)\right) \lambda_{1}\left\|u_{n}-\gamma_{n}^{\prime}\right\|\left\|A_{1} u_{n}-A_{1} q\right\| \\
& +2 \xi_{n} \alpha_{n}\left(1-\alpha_{n}\left(1-\sqrt{\frac{1-\delta}{\mu}}\right)\right)\left\|\gamma f\left(x_{n}\right)-A q\right\|\left\|y_{n}^{\prime}-q\right\| \\
& +\left(1-\xi_{n}\right)\left\|x_{n}-q\right\|^{2}-\left\|x_{n}-v_{n}\right\|^{2}+2\left(1-\xi_{n}\right) s_{n}\left\|B_{2} x_{n}-B_{2} q\right\|\left\|x_{n}-v_{n}\right\| \\
& -\left\|v_{n}-w_{n}\right\|^{2}+2\left(1-\xi_{n}\right) \lambda_{2}\left\|v_{n}-w_{n}\right\|\left\|A_{2} v_{n}-A_{2} q\right\| .
\end{aligned}
$$

Then, we derive

$$
\begin{aligned}
\| x_{n}- & u_{n}\left\|^{2}+\right\| u_{n}-y_{n}^{\prime}\left\|^{2}+\right\| x_{n}-v_{n}\left\|^{2}+\right\| v_{n}-w_{n} \|^{2} \\
\leq & \xi_{n} \alpha_{n}\left\|\gamma f\left(x_{n}\right)-A q\right\|^{2}+\left\|x_{n}-q\right\|^{2}-\left\|x_{n+1}-q\right\|^{2} \\
& +2 \xi_{n}\left(1-\alpha_{n}\left(1-\sqrt{\frac{1-\delta}{\mu}}\right)\right) r_{n}\left\|B_{1} x_{n}-B_{1} q\right\|\left\|x_{n}-u_{n}\right\| \\
& +2 \xi_{n}\left(1-\alpha_{n}\left(1-\sqrt{\frac{1-\delta}{\mu}}\right)\right) \lambda_{1}\left\|u_{n}-\gamma^{\prime}{ }_{n}\right\|\left\|A_{1} u_{n}-A_{1} q\right\| \\
& +2 \xi_{n} \alpha_{n}\left(1-\alpha_{n}\left(1-\sqrt{\frac{1-\delta}{\mu}}\right)\right)\left\|\gamma f\left(x_{n}\right)-A q\right\|\left\|y^{\prime}{ }_{n}-q\right\| \\
& +2\left(1-\xi_{n}\right) s_{n}\left\|B_{2} x_{n}-B_{2} q\right\|\left\|x_{n}-v_{n}\right\|+2\left(1-\xi_{n}\right) \lambda_{2}\left\|v_{n}-w_{n}\right\|\left\|A_{2} v_{n}-A_{2} q\right\| \\
= & \xi_{n} \alpha_{n}\left\|\lambda f\left(x_{n}\right)-A q\right\|^{2}+\left\|x_{n+1}-x_{n}\right\|\left(\left\|x_{n}-q\right\|+\left\|x_{n+1}-q\right\|\right) \\
& +2 \xi_{n}\left(1-\alpha_{n}\left(1-\sqrt{\frac{1-\delta}{\mu}}\right)\right) r_{n}\left\|B_{1} x_{n}-B_{1} q\right\|\left\|x_{n}-u_{n}\right\| \\
& +2 \xi_{n}\left(1-\alpha_{n}\left(1-\sqrt{\frac{1-\delta}{\mu}}\right)\right) \lambda_{1}\left\|u_{n}-\gamma^{\prime}\right\|\left\|A_{1} u_{n}-A_{1} q\right\| \\
& +2 \xi_{n} \alpha_{n}\left(1-\alpha_{n}\left(1-\sqrt{\frac{1-\delta}{\mu}}\right)\right)\left\|\gamma f\left(x_{n}\right)-A q\right\|\left\|y_{n}{ }_{n}-q\right\| \\
& +2\left(1-\xi_{n}\right) s_{n}\left\|B_{2} x_{n}-B_{2} q\right\|\left\|x_{n}-v_{n}\right\|+2\left(1-\xi_{n}\right) \lambda_{2}\left\|v_{n}-w_{n}\right\|\left\|A_{2} v_{n}-A_{2} q\right\| .
\end{aligned}
$$


By conditions (C1)-(C4), $\lim _{n \rightarrow \infty}|| x_{n}-x_{n+1}\left\|=0, \lim _{n \rightarrow \infty}|| B_{1} x_{n}-B_{1} q\right\|=0, \lim _{n \rightarrow \infty} \|$ $B_{2} x_{n}-B_{2} q\left\|=0, \lim _{n \rightarrow \infty}\right\| A_{1} u_{n}-A_{1} q\left\|=0, \lim _{n \rightarrow \infty}\right\| A_{2} v_{n}-A_{2} q\|=0,\| x_{n}-u_{n} \| \rightarrow 0$, $\left\|x_{n}-v_{n}\right\| \rightarrow 0,\left\|v_{n}-w_{n}\right\| \rightarrow 0$ as $n \rightarrow \infty$. So, we have $\left\|u_{n}-y_{n}^{\prime}\right\| \rightarrow 0$. It follows that

$$
\left\|x_{n}-w_{n}\right\| \leq\left\|x_{n}-v_{n}\right\|+\left\|v_{n}-w_{n}\right\| \rightarrow 0, \quad \text { as } n \rightarrow \infty .
$$

We compute that

$$
\begin{aligned}
\left\|x_{n+1}-x_{n}\right\| & =\left\|\xi_{n}\left(z_{n}-x_{n}\right)+\left(1-\xi_{n}\right)\left(P_{C} w_{n}-x_{n}\right)\right\| \\
& \leq \xi_{n}\left\|z_{n}-x_{n}\right\|+\left(1-\xi_{n}\right)\left\|w_{n}-x_{n}\right\| .
\end{aligned}
$$

From $\lim _{n \rightarrow \infty}|| x_{n}-w_{n}\left\|=0, \lim _{n \rightarrow \infty}|| x_{n+1}-x_{n}\right\|=0$, and hence

$$
\lim _{n \rightarrow \infty}\left\|z_{n}-x_{n}\right\|=0
$$

It follows by step 4 (i) and (ii),

$$
\left\|x_{n}-y_{n}\right\| \leq\left\|x_{n}-u_{n}\right\|+\left\|u_{n}-y_{n}\right\| \rightarrow 0, \quad \text { as } n \rightarrow \infty .
$$

Since

$$
\left\|z_{n}-y_{n}\right\| \leq\left\|z_{n}-x_{n}\right\|+\left\|x_{n}-y_{n}\right\| .
$$

So, by (3.41) and $\lim _{n \rightarrow \infty}|| x_{n}-y_{n}||=0$, we obtain

$$
\lim _{n \rightarrow \infty}\left\|z_{n}-y_{n}\right\|=0 .
$$

We show $\left\|S y^{\prime}{ }_{n}-z_{n}\right\| \rightarrow 0$ as $n \rightarrow \infty$. By nonexpansiveness of $P_{C}$ notice that

$$
\begin{aligned}
\left\|S y_{n}^{\prime}-z_{n}\right\| & =\left\|S y_{n}^{\prime}-P_{C}\left[\alpha_{n} \gamma f\left(x_{n}\right)+\left(I-\alpha_{n} A\right) S y_{n}^{\prime}\right]\right\| \\
& =\left\|P_{C} S y^{\prime}{ }_{n}-P_{C}\left[\alpha_{n} \gamma f\left(x_{n}\right)+\left(I-\alpha_{n} A\right) S y^{\prime}{ }_{n}\right]\right\| \\
& \leq\left\|S y^{\prime}{ }_{n}-\alpha_{n} \gamma f\left(x_{n}\right)-\left(I-\alpha_{n} A\right) S y^{\prime}{ }_{n}\right\| \\
& \leq \alpha_{n}\left\|\gamma f\left(x_{n}\right)-A S y^{\prime}{ }_{n}\right\| .
\end{aligned}
$$

By condition (C1), we get $\lim _{n \rightarrow \infty}\left\|S y^{\prime}{ }_{n}-z_{n}\right\|=0$. Since $\left\|S y^{\prime}{ }_{n}-y_{n}^{\prime}\right\| \leq\left\|S \gamma^{\prime}{ }_{n}-z_{n}\right\|+\left\|z_{n}-y_{n}\right\|+\left\|y_{n}-u_{n}\right\|+\left\|u_{n}-\gamma_{n}^{\prime}\right\|, \quad$ so $\quad$ by $\quad$ (3.42), $\lim _{n \rightarrow \infty}\left\|S y^{\prime}{ }_{n}-z_{n}\right\|=0, \lim _{n \rightarrow \infty}\left\|y_{n}-u_{n}\right\|=0$ and $\lim _{n \rightarrow \infty}\left\|u_{n}-y^{\prime}{ }_{n}\right\|=0$, we obtain $\lim _{n \rightarrow \infty}\left\|S y_{n}^{\prime}-\gamma_{n}^{\prime}\right\|=0$.

Step 5. We show that $q \in \Theta:=F(S) \cap \operatorname{GMEP}\left(F_{1}, \phi_{1}, B_{1}\right) \cap \operatorname{GMEP}\left(F_{2}, \phi_{2}, B_{2}\right) \cap I\left(A_{1}\right.$, $\left.M_{1}\right) \cap I\left(A_{2}, M_{2}\right)$ and $\lim \sup _{n \rightarrow \infty}\left\langle(\gamma f-A) q, S \gamma^{\prime}{ }_{n}-q\right\rangle \leq 0$. It is easy to see that $P_{\Theta}(\gamma f$ $+(I-A))$ is a contraction of $H$ into itself. In fact, from Lemma 2.9, we have

$$
\begin{aligned}
\left\|P_{\Theta}(\gamma f+(I-A)) x-P_{\Theta}(\gamma f+(I-A)) y\right\| & \leq\|(\gamma f+(I-A)) x-(\gamma f+(I-A)) \gamma\| \\
& \leq \gamma\|f(x)-f(\gamma)\|+(I-A)\|x-\gamma\| \\
& \leq \gamma \alpha\|x-\gamma\|+\left(1-\left(1-\sqrt{\frac{1-\delta}{\mu}}\right)\right)\|x-\gamma\| \\
& =\left(\sqrt{\frac{1-\delta}{\mu}}+\gamma \alpha\right)\|x-\gamma\| .
\end{aligned}
$$


Hence $H$ is complete, there exists a unique fixed point $q \in H$ such that $q=P_{\Theta}(\gamma f+$ $(I-A))(q)$. By Lemma 2.2 we obtain that $\langle(\gamma f-A) q, w-q\rangle \leq 0$ for all $w \in \Theta$.

Next, we show that $\lim \sup _{n \rightarrow \infty}\left\langle(\gamma f-A) q, S \gamma_{n}^{\prime}-q\right\rangle \leq 0$, where $q=P_{\Theta}(\gamma f+I-A)$ $(q)$ is the unique solution of the variational inequality $\langle(\not f-A) q, p-q\rangle \geq 0, \forall p \in \Theta$. We can choose a subsequence $\left\{\gamma_{n_{i}}^{\prime}\right\}$ of $\left\{\gamma_{n}^{\prime}\right\}$ such that

$$
\limsup _{n \rightarrow \infty}\left\langle(\gamma f-A) q, S \gamma_{n}^{\prime}-q\right\rangle=\lim _{i \rightarrow \infty}\left\langle(\gamma f-A) q, S y_{n_{i}}^{\prime}-q\right\rangle
$$

Since $\left\{y_{n_{i}}^{\prime}\right\}$ is bounded, there exists a subsequence $\left\{y_{n_{i}}^{\prime}\right\}$ of $\left\{y_{n_{i}}^{\prime}\right\}$ which converges weakly to $w$. We may assume without loss of generality that $y_{n_{i}}^{\prime} \rightarrow w$.

We claim that $w \in \Theta$. Since $\left\|y^{\prime}{ }_{n}-S y^{\prime}{ }_{n}\right\| \rightarrow 0$ and by Lemma 2.6, we have $w \in F(S)$.

Next, we show that $w \in \operatorname{GMEP}\left(F_{1}, \phi_{1}, B_{1}\right)$. Since $u_{n}=T_{r_{n}}^{\left(F_{1}, \varphi_{1}\right)}\left(x_{n}-r_{n} B_{1} x_{n}\right)$, we know that

$$
F_{1}\left(u_{n}, y\right)+\varphi_{1}(y)-\varphi_{1}\left(u_{n}\right)+\left\langle B_{1} x_{n}, y-u_{n}\right\rangle+\frac{1}{r_{n}}\left\langle y-u_{n}, u_{n}-x_{n}\right\rangle \geq 0, \quad \forall y \in C .
$$

It follows by (A2) that

$$
\varphi_{1}(y)-\varphi_{1}\left(u_{n}\right)+\left\langle B_{1} x_{n}, y-u_{n}\right\rangle+\frac{1}{r_{n}}\left\langle y-u_{n}, u_{n}-x_{n}\right\rangle \geq F_{1}\left(y, u_{n}\right), \quad \forall y \in C .
$$

Hence,

$$
\varphi_{1}(y)-\varphi_{1}\left(u_{n_{i}}\right)+\left\langle B_{1} x_{n_{i}}, y-u_{n_{i}}\right\rangle+\frac{1}{r_{n_{i}}}\left\langle y-u_{n_{i}}, u_{n_{i}}-x_{n_{i}}\right\rangle \geq F_{1}\left(y, u_{n_{i}}\right), \quad \forall y \in C .
$$

For $t \in(0,1]$ and $y \in H$, let $y_{t}=t y+(1-t) w$. From (3.43), we have

$$
\begin{aligned}
\left\langle y_{t}-u_{n_{i}}, B_{1} y_{t}\right\rangle \geq \geq & \left\langle y_{t}-u_{n_{i}}, B_{1} y_{t}\right\rangle-\varphi_{1}\left(y_{t}\right)+\varphi_{1}\left(u_{n_{i}}\right)-\left\langle B_{1} x_{n_{i}}, y_{t}-u_{n_{i}}\right\rangle \\
& -\frac{1}{r_{n_{i}}}\left\langle y_{t}-u_{n_{i^{\prime}}}, u_{n_{i}}-x_{n_{i}}\right\rangle+F_{1}\left(y_{t}, u_{n_{i}}\right) \\
= & \left\langle y_{t}-u_{n_{i}}, B_{1} y_{t}-B_{1} u_{n_{i}}\right\rangle+\left\langle y_{t}-u_{n_{i}}, B_{1} u_{n_{i}}-B_{1} x_{n_{i}}\right\rangle-\varphi_{1}\left(y_{t}\right)+\varphi_{1}\left(u_{n_{i}}\right) \\
& -\frac{1}{r_{n_{i}}}\left\langle y_{t}-u_{n_{i}}, u_{n_{i}}-x_{n_{i}}\right\rangle+F_{1}\left(y_{t}, u_{n_{i}}\right) .
\end{aligned}
$$

From $\left\|u_{n_{i}}-x_{n_{i}}\right\| \rightarrow 0$, we have $\left\|B_{1} u_{n_{i}}-B_{1} x_{n_{i}}\right\| \rightarrow 0$. Further, from (A4) and the weakly lower semicontinuity of $\varphi_{1}, \frac{u_{n_{i}}-x_{n_{i}}}{r_{n_{i}}} \rightarrow 0$ and $u_{n_{i}} \rightarrow w$, we have

$$
\left\langle y_{t}-w, B_{1} y_{t}\right\rangle \geq-\varphi_{1}\left(y_{t}\right)+\varphi_{1}(w)+F_{1}\left(y_{t}, w\right) .
$$


From (A1), (A4) and (3.44), we have

$$
\begin{aligned}
0 & =F_{1}\left(y_{t}, y_{t}\right)-\varphi_{1}\left(y_{t}\right)+\varphi_{1}\left(y_{t}\right) \\
& \leq t F_{1}\left(y_{t}, y\right)+(1-t) F_{1}\left(y_{t}, w\right)+t \varphi_{1}(y)+(1-t) \varphi_{1}(w)-\varphi_{1}\left(y_{t}\right) \\
& =t\left[F_{1}\left(y_{t}, y\right)+\varphi_{1}(y)-\varphi_{1}\left(y_{t}\right)\right]+(1-t)\left[F_{1}\left(y_{t}, w\right)+\varphi_{1}(w)-\varphi_{1}\left(y_{t}\right)\right] \\
& \leq t\left[F_{1}\left(y_{t}, y\right)+\varphi_{1}(y)-\varphi_{1}\left(y_{t}\right)\right]+(1-t)\left\langle y_{t}-w, B_{1} y_{t}\right\rangle \\
& =t\left[F_{1}\left(y_{t}, y\right)+\varphi_{1}(y)-\varphi_{1}\left(y_{t}\right)\right]+(1-t) t\left\langle y-w, B_{1} y_{t}\right\rangle,
\end{aligned}
$$

and hence

$$
0 \leq F_{1}\left(y_{t}, y\right)+\varphi_{1}(\gamma)-\varphi_{1}\left(y_{t}\right)+(1-t)\left\langle y-w, B_{1} y_{t}\right\rangle .
$$

Letting $t \rightarrow 0$, we have, for each $y \in C$,

$$
F_{1}(w, \gamma)+\varphi_{1}(\gamma)-\varphi_{1}(w)+\left\langle y-w, B_{1} w\right\rangle \geq 0 .
$$

This implies that $w \in \operatorname{GMEP}\left(F_{1}, \phi_{1}, B_{1}\right)$. By following the same arguments, we can show that $w \in \operatorname{GMEP}\left(F_{2}, \phi_{2}, B_{2}\right)$.

Lastly, we show that $w \in I\left(A_{1}, M_{2}\right)$. In fact, since $A_{1}$ is a $\beta_{1}$-inverse-strongly monotone, $A_{1}$ is monotone and Lipschitz continuous mapping. It follows from Lemma 2.3 that $M_{1}+A_{1}$ is a maximal monotone. Let $(v, g) \in G\left(M_{1}+A_{1}\right)$, since $g-A_{1} v \in M_{1}(v)$. Again since $y_{n_{i}}=J_{M_{1} \lambda_{1}}\left(u_{n_{i}}-\lambda_{1} A_{1} u_{n_{i}}\right)$, we have $u_{n_{i}}-\lambda_{1} A_{1} u_{n_{i}} \in\left(I+\lambda_{1} M_{1}\right)\left(y_{n_{i}}\right)$, that is, $\frac{1}{\lambda_{1}}\left(u_{n_{i}}-y_{n_{i}}-\lambda_{1} A_{1} u_{n_{i}}\right) \in M_{1}\left(y_{n_{i}}\right)$. By virtue of the maximal monotonicity of $M_{1}+$ $A_{1}$, we have

$$
\left\langle v-y_{n_{i}} g-A_{1} v-\frac{1}{\lambda_{1}}\left(u_{n_{i}}-y_{n_{i}}-\lambda_{1} A_{1} u_{n_{i}}\right)\right\rangle \geq 0,
$$

and hence

$$
\begin{aligned}
\left\langle v-y_{n_{i}}, g\right\rangle \geq & \left\langle v-y_{n_{i}}, A_{1} v+\frac{1}{\lambda}\left(u_{n_{i}}-y_{n_{i}}-\lambda_{1} A_{1} u_{n_{i}}\right)\right\rangle \\
= & \left\langle v-y_{n_{i}}, A_{1} v-A_{1} y_{n_{i}}\right\rangle+\left\langle v-y_{n_{i}}, A_{1} y_{n_{i}}-A_{1} u_{n_{i}}\right\rangle \\
& +\left\langle v-y_{n_{i}}, \frac{1}{\lambda_{1}}\left(u_{n_{i}}-y_{n_{i}}\right)\right\rangle .
\end{aligned}
$$

It follows from $\lim _{n \rightarrow \infty}|| u_{n}-y_{n}||=0$, we have $\lim _{n \rightarrow \infty}|| A_{1} u_{n}-A_{1} y_{n} \|=0$ and $y_{n_{i}} \rightarrow w$ that

$$
\limsup _{n \rightarrow \infty}\left\langle v-y_{n}, g\right\rangle=\langle v-w, g\rangle \geq 0 .
$$

It follows from the maximal monotonicity of $A_{1}+M_{1}$ that $\theta \in\left(M_{1}+A_{1}\right)(w)$, that is, $w \in I\left(A_{1}, M_{1}\right)$. By following the same arguments, we can show that $w \in I\left(A_{2}, M_{2}\right)$. Therefore, $w \in \Theta$. It follows that

$$
\limsup _{n \rightarrow \infty}\left\langle(\gamma f-A) q, S y^{\prime}{ }_{n}-q\right\rangle=\lim _{i \rightarrow \infty}\left\langle(\gamma f-A) q, S y_{n_{i}}^{\prime}-q\right\rangle=\langle(\gamma f-A) q, w-q\rangle \leq 0 .
$$

Step 6. We prove $x_{n} \rightarrow q$. By using (3.1), Lemma 2.9 (ii) and together with Schwarz inequality, we have 


$$
\begin{aligned}
& \left\|x_{n+1}-q\right\|^{2}=\left\|\xi_{n} P_{C}\left[\left(\alpha_{n} \gamma f\left(x_{n}\right)+\left(I-\alpha_{n} A\right) S y^{\prime}{ }_{n}\right)-q\right]+\left(1-\xi_{n}\right)\left(P_{C} w_{n}-q\right)\right\|^{2} \\
& \leq \xi_{n}\left\|P_{C}\left[\left(\alpha_{n} \gamma f\left(x_{n}\right)+\left(I-\alpha_{n} A\right) S y^{\prime}{ }_{n}\right)-P_{C}(q)\right]\right\|^{2}+\left(1-\xi_{n}\right)\left\|w_{n}-q\right\|^{2} \\
& \leq \xi_{n}\left\|\alpha_{n}\left(\gamma f\left(x_{n}\right)-A q\right)+\left(I-\alpha_{n} A\right)\left(S y^{\prime}{ }_{n}-q\right)\right\|^{2}+\left(1-\xi_{n}\right)\left\|x_{n}-q\right\|^{2} \\
& \leq \xi_{n}\left(I-\alpha_{n} A\right)^{2}\left\|S \gamma^{\prime}{ }_{n}-q\right\|^{2}+\xi_{n} \alpha_{n}^{2}\left\|\gamma f\left(x_{n}\right)-A q\right\|^{2} \\
& +2 \xi_{n} \alpha_{n}\left\langle\left(I-\alpha_{n} A\right)\left(S y_{n}^{\prime}-q\right), \gamma f\left(x_{n}\right)-A q\right\rangle+\left(1-\xi_{n}\right)\left\|x_{n}-q\right\|^{2} \\
& \leq \xi_{n}\left(1-\alpha_{n}\left(1-\sqrt{\frac{1-\delta}{\mu}}\right)\right)^{2}\left\|y_{n}-q\right\|^{2}+\xi_{n} \alpha_{n}^{2}\left\|\gamma f\left(x_{n}\right)-A q\right\|^{2} \\
& +2 \xi_{n} \alpha_{n}\left\langle S y_{n}^{\prime}-q, \gamma f\left(x_{n}\right)-A q\right\rangle \\
& -2 \xi_{n} \alpha_{n}^{2}\left\langle A\left(S y^{\prime}{ }_{n}-q\right), \gamma f\left(x_{n}\right)-A q\right\rangle+\left(1-\xi_{n}\right)\left\|x_{n}-q\right\|^{2} \\
& \leq \xi_{n}\left(1-\alpha_{n}\left(1-\sqrt{\frac{1-\delta}{\mu}}\right)\right)^{2}\left\|x_{n}-q\right\|^{2}+\xi_{n} \alpha_{n}^{2}\left\|\gamma f\left(x_{n}\right)-A q\right\|^{2} \\
& +2 \xi_{n} \alpha_{n}\left\langle S y_{n}^{\prime}-q, \gamma f\left(x_{n}\right)-\gamma f(q)\right\rangle+2 \xi_{n} \alpha_{n}\left\langle S y_{n}^{\prime}-q, \gamma f(q)-A q\right\rangle \\
& -2 \xi_{n} \alpha_{n}^{2}\left\langle A\left(S y_{n}^{\prime}-q\right), \gamma f\left(x_{n}\right)-A q\right\rangle+\left(1-\xi_{n}\right)\left\|x_{n}-q\right\|^{2} \\
& \leq \xi_{n}\left(1-\alpha_{n}\left(1-\sqrt{\frac{1-\delta}{\mu}}\right)\right)^{2}\left\|x_{n}-q\right\|^{2}+\xi_{n} \alpha_{n}^{2}\left\|\gamma f\left(x_{n}\right)-A q\right\|^{2} \\
& +2 \xi_{n} \alpha_{n}\left\|S \gamma_{n}^{\prime}-q\right\|\left\|\gamma f\left(x_{n}\right)-\gamma f(q)\right\|+2 \xi_{n} \alpha_{n}\left\langle S \gamma_{n}^{\prime}-q, \gamma f(q)-A q\right\rangle \\
& -2 \xi_{n} \alpha_{n}^{2}\left\langle A\left(S \gamma_{n}^{\prime}-q\right), \gamma f\left(x_{n}\right)-A q\right\rangle+\left(1-\xi_{n}\right)\left\|x_{n}-q\right\|^{2} \\
& \leq \xi_{n}\left(1-\alpha_{n}\left(1-\sqrt{\frac{1-\delta}{\mu}}\right)\right)^{2}\left\|x_{n}-q\right\|^{2}+\xi_{n} \alpha_{n}^{2}\left\|\gamma f\left(x_{n}\right)-A q\right\|^{2} \\
& +2 \xi_{n} \gamma \alpha \alpha_{n}\left\|y_{n}-q\right\|\left\|x_{n}-q\right\|+2 \xi_{n} \alpha_{n}\left\langle S y^{\prime}{ }_{n}-q, \gamma f(q)-A q\right\rangle \\
& -2 \xi_{n} \alpha_{n}^{2}\left\langle A\left(\gamma_{n}^{\prime}-q\right), \gamma f\left(x_{n}\right)-A q\right\rangle+\left(1-\xi_{n}\right)\left\|x_{n}-q\right\|^{2} \\
& \leq\left(\xi_{n}-2 \xi_{n} \alpha_{n}\left(1-\sqrt{\frac{1-\delta}{\mu}}\right)+\xi_{n} \alpha_{n}^{2}\left(1-\sqrt{\frac{1-\delta}{\mu}}\right)^{2}\right)\left\|x_{n}-q\right\|^{2} \\
& +\xi_{n} \alpha_{n}^{2}\left\|\gamma f\left(x_{n}\right)-A q\right\|^{2}+2 \xi_{n} \gamma \alpha \alpha_{n}\left\|x_{n}-q\right\|^{2} \\
& +2 \xi_{n} \alpha_{n}\left\langle S \gamma_{n}^{\prime}-q, \gamma f(q)-A q\right\rangle \\
& -2 \xi_{n} \alpha_{n}^{2}\left\langle A\left(S y^{\prime}{ }_{n}-q\right), \gamma f\left(x_{n}\right)-A q\right\rangle+\left(1-\xi_{n}\right)\left\|x_{n}-q\right\|^{2} \\
& \leq\left(1-2 \xi_{n} \alpha_{n}\left(1-\sqrt{\frac{1-\delta}{\mu}}\right)+2 \xi_{n} \gamma \alpha \alpha_{n}\right)\left\|x_{n}-q\right\|^{2}+\alpha_{n}\left\{\xi_{n} \alpha_{n}\left\|\gamma f\left(x_{n}\right)-A q\right\|^{2}\right. \\
& +2 \xi_{n}\left\langle S y_{n}^{\prime}-q, \gamma f(q)-A q\right\rangle-2 \xi_{n} \alpha_{n}\left\|A\left(S y_{n}^{\prime}-q\right)\right\|\left\|\gamma f\left(x_{n}\right)-A q\right\| \\
& \left.+\xi_{n} \alpha_{n}\left(1-\sqrt{\frac{1-\delta}{\mu}}\right)^{2}\left\|x_{n}-q\right\|^{2}\right\} \\
& =\left(1-2\left(\left(1-\sqrt{\frac{1-\delta}{\mu}}\right)-\gamma \alpha\right) \xi_{n} \alpha_{n}\right)\left\|x_{n}-q\right\|^{2}+\alpha_{n}\left\{\xi_{n} \alpha_{n}\left\|\gamma f\left(x_{n}\right)-A q\right\|^{2}\right. \\
& +2 \xi_{n}\left\langle S y_{n}^{\prime}-q, \gamma f(q)-A q\right\rangle-2 \xi_{n} \alpha_{n}\left\|A\left(S y_{n}^{\prime}-q\right)\right\|\left\|\gamma f\left(x_{n}\right)-A q\right\| \\
& \left.+\xi_{n} \alpha_{n}\left(1-\sqrt{\frac{1-\delta}{\mu}}\right)^{2}\left\|x_{n}-q\right\|^{2}\right\} \text {. }
\end{aligned}
$$

Since $\quad\left\{x_{n}\right\}$ is bounded, where $\eta \geq \xi_{n}\left\|\gamma f\left(x_{n}\right)-A q\right\|^{2}-2 \xi_{n}\left\|A\left(S y^{\prime}{ }_{n}-q\right)\right\|\left\|\gamma f\left(x_{n}\right)-A q\right\|+\xi_{n}\left(1-\sqrt{\frac{1-\delta}{\mu}}\right)^{2}\left\|x_{n}-q\right\|^{2}$ for all $n \geq$ 0. It follows that 


$$
\left\|x_{n+1}-q\right\|^{2} \leq\left(1-2\left(\left(1-\sqrt{\frac{1-\delta}{\mu}}\right)-\gamma \alpha\right) \xi_{n} \alpha_{n}\right)\left\|x_{n}-q\right\|^{2} \alpha_{n} \zeta_{n}
$$

where

$$
\varsigma_{n}=2 \xi_{n}\left\langle S \gamma_{n}^{\prime}-q, \gamma f(q)-A q\right\rangle+\eta \alpha_{n} .
$$

$\lim \sup _{n \rightarrow \infty}\left\langle(\gamma f-A) q, S \gamma^{\prime}{ }_{n}-q\right\rangle \leq 0$, we get $\lim \sup _{n \rightarrow \infty} \varsigma_{n} \leq 0$. Applying Lemma 2.5, we can conclude that $x_{n} \rightarrow q$. This completes the proof. $\square$

Next, the following example shows that all conditions of Theorem 3.1 are satisfied.

Example 3.2. For instance, let $\alpha_{n}=\frac{1}{2(n+1)}, \xi_{n}=\frac{2 n+2}{2(2 n)}, r_{n}=\frac{n}{n+1}$ and $s_{n}=\frac{n}{n+1}$, then, we will show that the sequences $\left\{\alpha_{n}\right\}$ satisfy the condition (C1). Indeed, we take $\alpha_{n}=\frac{1}{2(n+1)}$ then we have

$$
\begin{aligned}
& \lim _{n \rightarrow \infty} \alpha_{n}=\lim _{n \rightarrow \infty} \frac{1}{2(n+1)}=0 \\
& \sum_{n=1}^{\infty} \alpha_{n}=\sum_{n=1}^{\infty} \frac{1}{2(n+1)}=\infty
\end{aligned}
$$

and

$$
\sum_{n=1}^{\infty}\left|\alpha_{n}-\alpha_{n-1}\right|=\sum_{n=1}^{\infty}\left|\frac{1}{2(n+1)}-\frac{1}{2 n}\right| \leq\left|\frac{1}{2.2}-\frac{1}{2.1}\right|+\left|\frac{1}{2.3}-\frac{1}{2.2}\right|+\left|\frac{1}{2.4}-\frac{1}{2.3}\right|+\cdots=\frac{1}{2} .
$$

Then, the sequence $\left\{\alpha_{n}\right\}$ satisfy the condition (C1).

We will show that the sequences $\left\{\xi_{n}\right\}$ satisfy the condition (C2). Indeed, we set $\xi_{n}=\frac{2 n+2}{2(2 n)}=\frac{1}{2}+\frac{1}{2 n}$. By similarly $(\mathrm{C} 1)$, it is easy to see that $0<\lim \inf _{n \rightarrow \infty} \xi_{n}<\lim$ $\sup _{n \rightarrow \infty} \xi_{n}<1$ and $\sum_{n=1}^{\infty}\left|\xi_{n+1}-\xi_{n}\right|<\infty$.

Next, we will show that the condition (C3) is achieves. We take $r_{n}=\frac{n}{n+1}$, then we compute

$$
\begin{aligned}
\sum_{n=1}^{\infty}\left|r_{n}-r_{n-1}\right| & =\sum_{n=1}^{\infty}\left|\frac{n}{n+1}-\frac{n-1}{(n-1)+1}\right|=\sum_{n=1}^{\infty}\left|\frac{n(n)-(n-1)(n+1)}{(n+1) n}\right| \\
& =\sum_{n=1}^{\infty}\left|\frac{n^{2}-n^{2}+1}{(n+1) n}\right|=\sum_{n=1}^{\infty}\left|\frac{1}{n(n+1)}\right| .
\end{aligned}
$$

Then, we have $\liminf _{n \rightarrow \infty} r_{n}=\liminf _{n \rightarrow \infty} \frac{n}{n+1}=1$ and also $\lim _{n \rightarrow \infty}\left|r_{n+1}-r_{n}\right|=0$.

The sequence $\left\{r_{n}\right\}$ satisfy the condition (C3). In the same way with (C4). $\square$

Corollary 3.3. Let $H$ be a real Hilbert space, $C$ be a closed convex subset of $H$. Let $F_{1}$, $F_{2}$ be bifunctions of $C \times C$ into $\mathcal{R}$ satisfying (A1)-(A4) and $B, B_{1}, B_{2}: C \rightarrow H$ be $\beta$, $\eta$, $\rho$-inverse-strongly monotone mappings, $\varphi_{1}, \varphi_{2}: C \rightarrow \mathcal{R}$ be convex and lower semicontinuous functions, $f: C \rightarrow C$ be a contraction with coefficient $\alpha(0<\alpha<1), M: H \rightarrow$ $2^{H}$ be a maximal monotone mapping. Assume that either (B1) or (B2) holds. Let $S$ be a nonexpansive mapping of $C$ into itself such that 


$$
\Theta_{1}:=F(S) \cap \operatorname{GMEP}\left(F_{1}, \varphi_{1}, B_{1}\right) \cap \operatorname{GMEP}\left(F_{2}, \varphi_{2}, B_{2}\right) \cap I(B, M) \neq \emptyset .
$$

Suppose $\left\{x_{n}\right\}$ is a sequences generated by the following algorithm $x_{0} \in C$ arbitrarily:

$$
\left\{\begin{array}{l}
u_{n}=T_{r_{n}}^{\left(F_{1}, \varphi_{1}\right)}\left(x_{n}-r_{n} B_{1} x_{n}\right) \\
v_{n}=T_{\left.s_{n}, \varphi_{2}\right)}^{\left(F_{2}\right)}\left(x_{n}-s_{n} B_{2} x_{n}\right) \\
x_{n+1}=\xi_{n} P_{C}\left[\alpha_{n} f\left(x_{n}\right)+\left(I-\alpha_{n}\right) S P_{C}\left(J_{M, \lambda}(I-\lambda B) u_{n}\right)\right]+\left(1-\xi_{n}\right) P_{C} v_{n}
\end{array}\right.
$$

where $\left\{\alpha_{n}\right\},\left\{\xi_{n}\right\} \subset(0,1), \lambda \in(0,2 \beta)$ such that $0<a \leq \lambda \leq b<2 \beta, r_{n} \in(0,2 \eta)$ with 0 $<c \leq d \leq 1-\eta$ and $s_{n} \in(0,2 \rho)$ with $0<e \leq f \leq 1-\rho$ satisfy the following conditions:

(C1): $\lim _{n \rightarrow \infty} \alpha_{n}=0, \sum_{n=0}^{\infty} \alpha_{n}=\infty, \sum_{n=1}^{\infty}\left|\alpha_{n+1}-\alpha_{n}\right|<\infty$,

(C2): $0<\lim _{\inf _{n \rightarrow \infty}} \xi_{n}<\lim \sup _{n \rightarrow \infty} \xi_{n}<1, \sum_{n=1}^{\infty}\left|\xi_{n+1}-\xi_{n}\right|<\infty$,

(C3): $\lim \inf _{n \rightarrow \infty} r_{n}>0$ and $\lim _{n \rightarrow \infty}\left|r_{n+1}-r_{n}\right|=0$,

(C4): $\lim \inf _{n \rightarrow \infty} s_{n}>0$ and $\lim _{n \rightarrow \infty}\left|s_{n+1}-s_{n}\right|=0$.

Then $\left\{x_{n}\right\}$ converges strongly to $q \in \Theta_{1}$, where $q=P_{\Theta_{1}}(f+I)(q)$ which solves the following variational inequality:

$$
\langle(f-I) q, p-q\rangle \leq 0, \quad \forall p \in \Theta_{1} .
$$

Proof. Putting $A \equiv I, \gamma \equiv 1, J_{\mathrm{M}_{2}, \lambda_{2}} \equiv I$ and $A_{2} \equiv 0$ in Theorem 3.1, we can obtain desired conclusion immediately.

Corollary 3.4. Let $H$ be a real Hilbert space, $C$ be a closed convex subset of $H$. Let $F_{1}$, $F_{2}$ be bifunctions of $C \times C$ into $\mathcal{R}$ satisfying (A1)-(A4) and $B, B_{1}, B_{2}: C \rightarrow H$ be $\beta, \eta, \rho$ inverse-strongly monotone mappings, $\varphi_{1}, \varphi_{2}: C \rightarrow \mathcal{R}$ be convex and lower semicontinuous functions. Assume that either (B1)or (B2) holds. Let $S$ be a nonexpansive mapping of $C$ into itself such that

$$
\Theta_{1}:=F(S) \cap \operatorname{GMEP}\left(F_{1}, \varphi_{1}, B_{1}\right) \cap \operatorname{GMEP}\left(F_{2}, \varphi_{2}, B_{2}\right) \cap I(B, M) \neq \emptyset .
$$

Suppose $\left\{x_{n}\right\}$ is a sequences generated by the following algorithm $x_{0} \in C$ arbitrarily:

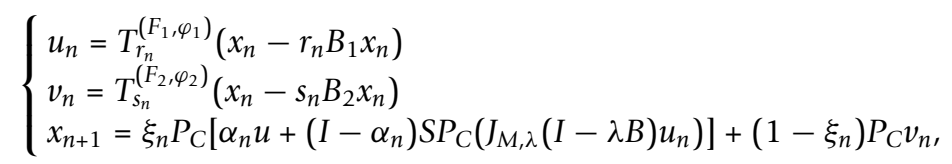

where $\left\{\alpha_{n}\right\},\left\{\xi_{n}\right\} \subset(0,1), \lambda \in(0,2 \beta)$ such that $0<a \leq \lambda \leq b<2 \beta, r_{n} \in(0,2 \eta)$ with 0 $<c \leq d \leq 1-\eta$ and $s_{n} \in(0,2 \rho)$ with $0<e \leq f \leq 1-\rho$ satisfy the following conditions:

(C1): $\lim _{n \rightarrow \infty} \alpha_{n}=0, \sum_{n=0}^{\infty} \alpha_{n}=\infty, \sum_{n=1}^{\infty}\left|\alpha_{n+1}-\alpha_{n}\right|<\infty$,

(C2): $0<\lim \inf _{n \rightarrow \infty} \xi_{n}<\lim \sup _{n \rightarrow \infty} \xi_{n}<1, \sum_{n=1}^{\infty}\left|\xi_{n+1}-\xi_{n}\right|<\infty$,

(C3): $\lim \inf _{n \rightarrow \infty} r_{n}>0$ and $\lim _{n \rightarrow \infty}\left|r_{n+1}-r_{n}\right|=0$,

(C4): $\lim \inf _{n \rightarrow \infty} s_{n}>0$ and $\lim _{n \rightarrow \infty}\left|s_{n+1}-s_{n}\right|=0$.

Then $\left\{x_{n}\right\}$ converges strongly to $q \in \Theta_{1}$, where $q=P_{\Theta_{1}}(q)$ which solves the following variational inequality:

$$
\langle u-q, p-q\rangle \leq 0, \quad \forall p \in \Theta_{1} .
$$

Proof. Putting $f \equiv u \in C$ in Corollary 3.3, we can obtain desired conclusion immediately. $\square$ 
Corollary 3.5. Let $H$ be a real Hilbert space, $C$ be a closed convex subset of $H$. Let $F_{1}$, $F_{2}$ be bifunctions of $C \times C$ into $\mathcal{R}$ satisfying (A1)-(A4) and $B, B_{1}, B_{2}: C \rightarrow H$ be $\beta, \eta$, $\rho$ inverse-strongly monotone mappings, $\varphi_{1}, \varphi_{2}: C \rightarrow \mathcal{R}$ be convex and lower semicontinuous functions, $f: C \rightarrow C$ be a contraction with coefficient $\alpha(0<\alpha<1)$ and $A$ is a $\delta$ strongly monotone and $\mu$-strictly pseudo-contraction with $\delta+\mu>1, \gamma$ is a positive real number such that $\gamma<\frac{1}{\alpha}\left(1-\sqrt{\frac{1-\delta}{\mu}}\right)$. Assume that either (B1) or (B2) holds. Let $S$ be a nonexpansive mapping of $C$ into itself such that

$$
\Theta_{2}:=F(S) \cap \operatorname{GMEP}\left(F_{1}, \varphi_{1}, B_{1}\right) \cap \operatorname{GMEP}\left(F_{2}, \varphi_{2}, B_{2}\right) \cap V I(C, B) \neq \emptyset .
$$

Suppose $\left\{x_{n}\right\}$ is a sequences generated by the following algorithm $x_{0} \in C$ arbitrarily:

$$
\left\{\begin{array}{l}
u_{n}=T_{r_{n}}^{\left(F_{1}, \varphi_{1}\right)}\left(x_{n}-r_{n} B_{1} x_{n}\right) \\
v_{n}=T_{s_{n}}^{\left(F_{2}, \varphi_{2}\right)}\left(x_{n}-s_{n} B_{2} x_{n}\right) \\
x_{n+1}=\xi_{n} P_{C}\left[\alpha_{n} \gamma f\left(x_{n}\right)+\left(I-\alpha_{n} A\right) S P_{C}(I-\lambda B) u_{n}\right]+\left(1-\xi_{n}\right) P_{C} v_{n}
\end{array}\right.
$$

where $\left\{\alpha_{n}\right\},\left\{\xi_{n}\right\} \subset(0,1), \lambda \subset(0,2 \beta)$ such that $0<a \leq \lambda \leq b<2 \beta, r_{n} \in(0,2 \eta)$ with 0 $<c \leq d \leq 1-\eta$ and $s_{n} \in(0,2 \rho)$ with $0<e \leq f \leq 1-\rho$ satisfy the following conditions:

(C1): $\lim _{n \rightarrow \infty} \alpha_{n}=0, \sum_{n=0}^{\infty} \alpha_{n}=\infty, \sum_{n=1}^{\infty}\left|\alpha_{n+1}-\alpha_{n}\right|<\infty$,

(C2): $0<\lim \inf _{n \rightarrow \infty} \xi_{n}<\lim \sup _{n \rightarrow \infty} \xi_{n}<1, \sum_{n=1}^{\infty}\left|\xi_{n+1}-\xi_{n}\right|<\infty$,

(C3): $\lim _{\inf _{n \rightarrow \infty}} r_{n}>0$ and $\lim _{n \rightarrow \infty}\left|r_{n+1}-r_{n}\right|=0$,

(C4): $\lim _{\inf _{n \rightarrow \infty}} s_{n}>0$ and $\lim _{n \rightarrow \infty}\left|s_{n+1}-s_{n}\right|=0$.

Then $\left\{x_{n}\right\}$ converges strongly to $q \in \Theta_{2}$, where $q=P_{\Theta_{2}}(\gamma f+I-A)(q)$ which solves the following variational inequality:

$$
\langle(\gamma f-A) q, p-q\rangle \leq 0, \quad \forall p \in \Theta_{2} .
$$

Proof. Taking $J_{M_{1}, \lambda_{1}} \equiv I, J_{M_{2}, \lambda_{2}} \equiv I, A_{1} \equiv B$ and $A_{2} \equiv 0$ in Theorem 3.1, we can obtain desired conclusion immediately.

Corollary 3.6. Let $H$ be a real Hilbert space, $C$ be a closed convex subset of $H$. Let $F_{1}$, $F_{2}$ be bifunctions of $C \times C$ into $\mathcal{R}$ satisfying (A1)-(A4) and $B_{1}, B_{2}: C \rightarrow H$ be $\eta, \rho$ inverse-strongly monotone mappings, $\varphi_{1}, \varphi_{2}: C \rightarrow \mathcal{R}$ be convex and lower semicontinuous functions, $f: C \rightarrow C$ be a contraction with coefficient $\alpha(0<\alpha<1)$. Assume that either (B1) or (B2) holds. Let $S$ be a nonexpansive mapping of $C$ into itself such that

$$
\Theta_{3}:=F(S) \cap \operatorname{GMEP}\left(F_{1}, \varphi_{1}, B_{1}\right) \cap \operatorname{GMEP}\left(F_{2}, \varphi_{2}, B_{2}\right) \neq \emptyset .
$$

Suppose $\left\{x_{n}\right\}$ is a sequences generated by the following algorithm $x_{0} \in C$ arbitrarily:

$$
\left\{\begin{array}{l}
u_{n}=T_{r_{n}}^{\left(F_{1}, \varphi_{1}\right)}\left(x_{n}-r_{n} B_{1} x_{n}\right) \\
v_{n}=T_{\left.s_{n}, \varphi_{2}\right)}^{\left(F_{2}\right.}\left(x_{n}-s_{n} B_{2} x_{n}\right) \\
x_{n+1}=\xi_{n} P_{C}\left[\alpha_{n} f\left(x_{n}\right)+\left(I-\alpha_{n}\right) S P_{C} u_{n}\right]+\left(1-\xi_{n}\right) P_{C} v_{n}
\end{array}\right.
$$

where $\left\{\alpha_{n}\right\},\left\{\xi_{n}\right\} \subset(0,1), r_{n} \in(0,2 \eta)$ with $0<c \leq d \leq 1-\eta$ and $s_{n} \in(0,2 \rho)$ with $0<e$ $\leq f \leq 1-\rho$ satisfy the following conditions:

(C1): $\lim _{n \rightarrow \infty} \alpha_{n}=0, \sum_{n=0}^{\infty} \alpha_{n}=\infty, \sum_{n=1}^{\infty}\left|\alpha_{n+1}-\alpha_{n}\right|<\infty$,

(C2): $0<\lim _{\inf _{n \rightarrow \infty}} \xi_{n}<\lim \sup _{n \rightarrow \infty} \xi_{n}<1, \sum_{n=1}^{\infty}\left|\xi_{n+1}-\xi_{n}\right|<\infty$,

(C3): $\lim \inf _{n \rightarrow \infty} r_{n}>0$ and $\lim _{n \rightarrow \infty}\left|r_{n+1}-r_{n}\right|=0$, 
(C4): $\lim \inf _{n \rightarrow \infty} s_{n}>0$ and $\lim _{n \rightarrow \infty}\left|s_{n+1}-s_{n}\right|=0$.

Then $\left\{x_{n}\right\}$ converges strongly to $q \in \Theta_{3}$, where $q=P_{\Theta_{3}}(f+I)(q)$ which solves the following variational inequality:

$$
\langle(f-I) q, p-q\rangle \leq 0, \quad \forall p \in \Theta_{3}
$$

Proof. Taking $\gamma \equiv 1, A \equiv I$ and $B \equiv 0$ in Corollary 3.5, we can obtain desired conclusion immediately.

\section{Acknowledgements}

The authors are grateful for the reviewers for the careful reading of the article and for the suggestions which improved the quality of this work.

This research was partially supported by the Hands-On Research and Development, Rajamangala University of Technology Lanna (under Project No. UR2L-003). Furthermore, the third authors also would like to thank the Higher Education Research Promotion and National Research University Project of Thailand, Office of the Higher Education Commission (NRU-CSEC Project No.55000613) for financial support.

\section{Author details}

${ }^{1}$ Department of Mathematics and Statistics, Faculty of Science and Agricultural Technology, Rajamangala University of Technology Lanna Tak, Tak 63000, Thailand ²Department of Mathematics, Faculty of Science, King Mongkut's University of Technology (KMUTT), Bangmod, Thrungkru, Bangkok 10140, Thailand

\section{Authors' contributions}

All authors contributed equally and significantly in writing this article. All authors read and approved the final manuscript.

\section{Competing interests}

The authors declare that they have no competing interests.

Received: 6 March 2012 Accepted: 13 June 2012 Published: 13 June 2012

\section{References}

1. Browder, FE: Existence and approximation of solutions of nonlinear variational inequalities. Proc Natl Acad Sci USA. 56, 1080-1086 (1966). doi:10.1073/pnas.56.4.1080

2. Blum, E, Oettli, W: From optimization and variational inequalities to equilibrium problems. Math Student. 63, 123-145 (1994)

3. Chang, SS, Lee, HWJ, Chan, CK: A new method for solving equilibrium problem fixed point problem and variational inequality problem with application to optimization. Nonlinear Anal Theory Method Appl. 670, 3307-3319 (2009)

4. Shehu, Y: A new iterative scheme for a countable family of relatively nonex-pansive mappings and an equilibrium problem in Banach spaces. J Global Optim

5. $\mathrm{Ye}_{\mathrm{e}} \mathrm{J}$, Huang, J: Strong convergence theorems for fixed point problems and generalized equilibrium problems of three relatively quasi-nonexpansive mappings in Banach spaces. J Math Comput Sci. 1, 1-18 (2011)

6. Yang, S, Li, W: Iterative solutions of a system of equilibrium problems in Hilbert spaces. Adv Fixed Point Theory. 1, $15-26(2011)$

7. Hartman, P, Stampacchia, G: On some nonlinear elliptic differential functional equations. Acta Math. 115, 271-310 (1966). doi:10.1007/BF02392210

8. Marino, G, Xu, HK: A general iterative method for nonexpansive mapping in Hilbert space. J Math Anal Appl. 318 43-52 (2006). doi:10.1016/j.jmaa.2005.05.028

9. Kirk, WA: Fixed point theorem for mappings which do not increase distance. Am Math Monthly. 72, $1004-1006$ (1965). doi:10.2307/2313345

10. Browder, FE, Petryshym, WV: Construction of fixed points of nonlinear mappings in Hilbert spaces. J Math Anal Appl. 20, 197-228 (1963)

11. Liu, M, Chang, SS, Zuo, P: An algorithm for finding a common solution for a system of mixed equilibrium problem, quasivariational inclusion problems of nonexpansive semigroup. J Inequal Appl 23 (2010). Article ID 895907,

12. Hao, Y: Some results of variatinal inclusion problems and fixed point problems with applications. Appl Math Mech. 30(12), 1589-1596 (2009). doi:10.1007/s10483-009-1210-x

13. Matsushita, SY, Takahashi, W: Existence theorems for set-valued operators in Banach spaces. Set-Valued Anal. 15 251-264 (2007). doi:10.1007/s11228-006-0030-8

14. Tan, JF, Chang, SS: Iterative algorithms for finding common solutions to variational inclusion equilibrium and fixed point problems. Fixed Point Theory Appl 17 (2011). Article ID 915629,

15. Zhang, SS, Joseph Lee, HW, Chan, CK: Algorithms of common solutions to quasi variational inclusion and fixed point problems. Appl Math Mech. 29(5) (2008)

16. Rockafellar, RT: On the maximality of sums of nonlinear monotone operators. Trans Am Math Soc. 149, 46-55 (2000)

17. Takahashi, W: Nonlinear Functional Analysis. Yokohama Publishers, Yokohama (2000)

18. Moudafi, A: Viscosity approximation methods for fixed points problems. J Math Anal Appl. 241, 46-55 (2000). doi:10.1006/jmaa.1999.6615 
19. liduka, $\mathrm{H}$, Takahashi, W: Strong convergence theorems for nonexpansive mapping and inverse-strong monotone mappings. Nonlinear Anal. 61, 341-350 (2005). doi:10.1016/j.na.2003.07.023

20. Su, Y, Shang, M, Qin, X: An iterative method of solution for equilibrium and optimization problems. Nonlinear Anal. 69 , 2709-2719 (2008). doi:10.1016/j.na.2007.08.045

21. Brézis, H: Opérateur maximaux monotones. In Mathematics Studies, vol. 5,North-Holland, Amsterdam (1973)

22. Opial, Z: Weak convergence of successive approximations for nonexpansive mappings. Bull Am Math Soc. 73, 591-597 (1967). doi:10.1090/S0002-9904-1967-11761-0

23. Xu, HK: Iterative algorithms for nonlinear operators. J Lond Math Soc. 66, 240-256 (2002). doi:10.1112/ S0024610702003332

24. Browder, FE: Nonlinear operators and nonlinear equations of evolution in Banach spaces. Proc Symp Pure Math. 18 78-81 (1976)

25. Peng, J-W, Liou, Y-C, Yao, J-C: An iterative algorithm combining viscosity method with parallel method for a generalized equilibrium problem and strict pseudocontractions. Fixed Point Theory Appl 21 (2009). Article ID 794178 ,

26. Wangkeeree, R, Bantaojai, T: A general composite algorithms for solving general equilibrium problems and fixed point problems in Hilbert spaces. Thai J Math. 9, 191-212 (2011)

doi:10.1186/1029-242X-2012-138

Cite this article as: Wattanawitoon et al: A new hybrid general iterative algorithm for common solutions of generalized mixed equilibrium problems and variational inclusions. Journal of Inequalities and Applications 2012 2012:138

\section{Submit your manuscript to a SpringerOpen ${ }^{\circ}$} journal and benefit from:

- Convenient online submission

- Rigorous peer review

- Immediate publication on acceptance

- Open access: articles freely available online

- High visibility within the field

- Retaining the copyright to your article

Submit your next manuscript at $\gg$ springeropen.com 